\title{
Feedback Amplitude Modulation Synthesis
}

\author{
Jari Kleimola, ${ }^{1}$ Victor Lazzarini, ${ }^{2}$ Vesa Välimäki, ${ }^{1}$ and Joseph Timoney ${ }^{2}$ \\ ${ }^{1}$ Department of Signal Processing and Acoustics, Aalto University School of Electrical Engineering, P.O. Box 13000, \\ 00076 AALTO, Espoo, Finland \\ ${ }^{2}$ Sound and Digital Music Technology Group, National University of Ireland, Maynooth, Co. Kildare, Ireland
}

Correspondence should be addressed to Jari Kleimola, jari.kleimola@tkk.fi

Received 15 September 2010; Accepted 20 December 2010

Academic Editor: Federico Fontana

Copyright () 2011 Jari Kleimola et al. This is an open access article distributed under the Creative Commons Attribution License, which permits unrestricted use, distribution, and reproduction in any medium, provided the original work is properly cited.

A recently rediscovered sound synthesis method, which is based on feedback amplitude modulation (FBAM), is investigated. The FBAM system is interpreted as a periodically linear time-varying digital filter, and its stability, aliasing, and scaling properties are considered. Several novel variations of the basic system are derived and analyzed. Separation of the input and the modulation signals in FBAM structures is proposed which helps to create modular sound synthesis and digital audio effects applications. The FBAM is shown to be a powerful and versatile sound synthesis principle, which has similarities to the established distortion synthesis methods, but which is also essentially different from them.

\section{Introduction}

Amplitude modulation (AM) is a well-described technique of sound processing [1]. It is based on the audio-range modulation of the amplitude of a carrier signal generator by another signal. For each component in the two input signals, three components will be produced at the output: the sum and difference between the two, plus the carrier signal component. The amplitude of the output signal $s_{\mathrm{AM}}(n)$ is offset by the carrier amplitude $a$, that is,

$$
s_{\mathrm{AM}}(n)=\left[s_{m}(n)+a\right] \frac{s_{c}(n)}{a}
$$

where $s_{c}(n)$ and $s_{m}(n)$ are the carrier and modulation signals, respectively, and $a$ is the maximum absolute amplitude of the carrier signal.

$\mathrm{AM}$ has a sister technique, ring modulation (RM) [1], which is very similar, but with one important difference: there is no offset in the output amplitude, and the output signal can be expressed as

$$
s_{\mathrm{RM}}(n)=s_{m}(n) s_{c}(n) .
$$

Thus, the spectrum of ring modulation will not contain the carrier signal.
For sinusoidal inputs, both techniques will produce a limited set of partials. In order to develop them into a useful method of synthesis, one may either employ a componentrich carrier, or by means of feedback, add partials to the modulator [2]. The second option has the advantage of providing a rich output simply using two sinusoidal oscillators. Note that in this case only the AM method is practical, since feedback RM produces only silence after the modulator signal becomes zero.

The feedback AM (FBAM) oscillator first appeared in the literature as instrument 1 in example no. 510 from Risset's catalogue of computer synthesized sounds [3] and subsequently in a conference paper by Layzer [4] to whom Risset had attributed the idea. Also, a further implementation of the algorithm is found in [5].

However, the FBAM algorithm remains relatively unknown and, apart from the prior work cited above, is largely unexplored. The authors started examining it in [2] and will now expand this work in order to provide a framework for a general theory of feedback synthesis by exploring the periodically linear time-variant (PLTV) filter theory in synthesis contexts. A further goal is to gain a better understanding of FBAM for practical implementation purposes. The novel work comprises (i) the PLTV filter interpretation of the method, (ii) stability, aliasing, and scaling considerations, 


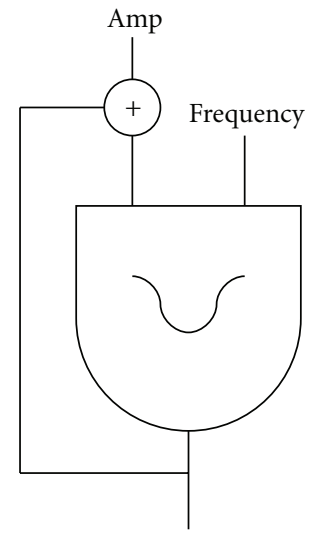

Figure 1: Feedback AM oscillator [4].

(iii) detailed analysis of the variations, (iv) additional variations and implementations (generalized coefficientmodulated IIR filter, adaptive FBAM, Csound opcode), and (v) evaluation and applications of the FBAM method.

The paper is organized as follows. Section 2 presents the basic FBAM structure and contextualizes it as a coefficientmodulated first-order feedback filter. Section 3 proposes six general variations on the basic equation, while Section 4 explores the implementation aspects of FBAM in the form of synthesis operator structures. Section 5 evaluates the FBAM method against established nonlinear distortion techniques, Section 6 discusses its applications in various areas of digital sound generation and effects, and, finally, Section 7 concludes.

\section{Feedback AM Oscillator}

The signal flowchart of Layzer's feedback AM instrument is shown in Figure 1. This instrument is now investigated in detail by interpreting it as a periodically linear time-variant filter. The basic FBAM equation with feedback amount control is then introduced, and its impact on the stability, aliasing, and scaling properties of the system is discussed.

2.1. The FBAM Algorithm. First, consider the simplest FBAM form, utilizing a unit delay feedback, that can be written as

$$
y(n)=\cos \left(\omega_{0} n\right)[1+y(n-1)]
$$

with the fundamental frequency $f_{0}$, the sampling rate $f_{s}$, and $\omega_{0}=2 \pi f_{0} / f_{s}$. The initial condition $y(n)=0$, for $n \leq 0$, is used in this and all other recursive equations in this paper. This feedback expression can be expanded into an infinite sum of products given by

$$
\begin{aligned}
y(n)= & \cos \left(\omega_{0} n\right)+\cos \left(\omega_{0} n\right) \cos \left(\omega_{0}[n-1]\right) \\
& +\cos \left(\omega_{0} n\right) \cos \left(\omega_{0}[n-1]\right) \cos \left(\omega_{0}[n-2]\right)+\cdots \\
= & \sum_{k=0}^{\infty} \prod_{m=0}^{k} \cos \left[\omega_{0}(n-m)\right],
\end{aligned}
$$

which leads to the conclusion that the resulting spectrum is composed of various harmonics of the fundamental $f_{0}$. In fact, as can be seen in Figure 2, a smooth pulse-like waveform that reaches its steady-state condition within the first period of the waveform is obtained (the reduced initial peak of the waveform is not present if the $\cos (\cdot)$ term of (3) is replaced by a $\sin (\cdot)$ term. The cosine form, however, simplifies the theoretical discussion).

Rewriting (4) as

$$
y(n)=\sum_{k=0}^{\infty} \frac{p_{k}}{2^{k}}
$$

with

$$
p_{k}=2^{k} \prod_{m=0}^{k} \cos \left[\omega_{0}(n-m)\right],
$$

one gets a glimpse of what the resulting spectrum might look like. The products $p_{k}$ for $k=0 \cdots 4$ are the following:

$$
\begin{aligned}
p_{0}= & \cos \left(\omega_{0} n\right) \\
p_{1}= & \cos \left(\omega_{0}\right)+\cos \left[2 \omega_{0}\left(n-\frac{1}{2}\right)\right] \\
p_{2}= & \cos \left[\omega_{0}(n-3)\right]+2 \cos \left(\omega_{0}\right) \cos \left(\omega_{0} n\right)+\cos \left[3 \omega_{0}(n-1)\right], \\
p_{3}= & +\cos \left(2 \omega_{0}\right)+\cos \left(4 \omega_{0}\right)+\cos \left[2 \omega_{0}\left(n-\frac{3}{2}\right)\right] \\
& +\cos \left[2 \omega_{0}(n-2)\right]+\cos \left[2 \omega_{0}(n-1)\right]+\cos \left(2 \omega_{0} n\right) \\
& +\cos \left[4 \omega_{0}\left(n-\frac{3}{2}\right)\right], \\
p_{4}= & \cos \left[\omega_{0}(n-8)\right]+\cos \left[\omega_{0}(n-6)\right]+\cos \left[\omega_{0}(n-2)\right] \\
& +2 \cos \left(4 \omega_{0}\right) \cos \left(\omega_{0} n\right)+2 \cos \left(2 \omega_{0}\right) \cos \left(\omega_{0} n\right) \\
& +\cos \left[3 \omega_{0}\left(n-\frac{10}{3}\right)\right]+\cos \left[3 \omega_{0}\left(n-\frac{8}{3}\right)\right] \\
& +\cos \left[3 \omega_{0}(n-2)\right]+\cos \left[3 \omega_{0}\left(n-\frac{4}{3}\right)\right] \\
& +\cos \left[3 \omega_{0}\left(n-\frac{3}{2}\right)\right]+\cos \left[5 \omega_{0}(n-2)\right] .
\end{aligned}
$$

So, for this partial sum, the fundamental (harmonic 1 ) is a combination of cosines having slightly different phases and amplitudes

$$
\begin{aligned}
& \frac{1}{16}\left\{\cos \left[\omega_{0}(n-8)\right]+\cos \left[\omega_{0}(n-6)\right]\right. \\
& \left.\quad+\cos \left[\omega_{0}(n-2)\right]\right\}+\frac{1}{4} \cos \left[\omega_{0}(n-3)\right] \\
& +\left\{\frac{1}{2} \cos \left(\omega_{0}\right)+\frac{1}{8}\left[\cos \left(4 \omega_{0}\right)+\cos \left(2 \omega_{0}\right)\right]+1\right\} \cos \left(\omega_{0} n\right) .
\end{aligned}
$$

This indicates that the harmonic amplitudes will be dependent on the fundamental frequency (given the various $\cos (\cdot)$ 


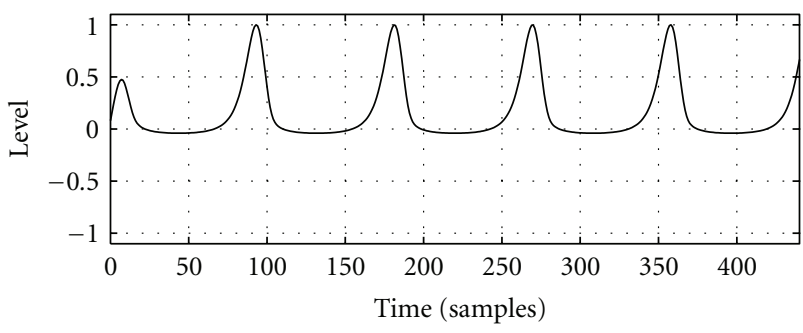

(a)

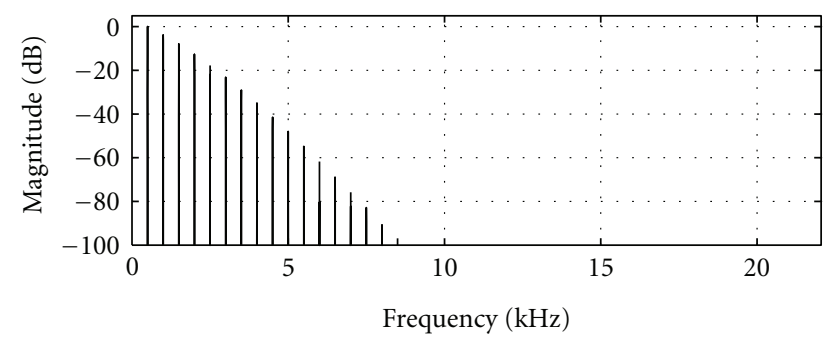

(b)

Figure 2: Peak-normalized FBAM waveform $\left(f_{0}=500 \mathrm{~Hz}\right)$ and its spectrum. The sample rate $f_{s}=44.1 \mathrm{kHz}$ is used in this and all other examples in this paper unless noted otherwise.

terms in the scaling of some components). The combined magnitudes of the components will also depend on the fundamental frequency and sampling rate because of the mixture of various delayed terms.

Figure 2 shows that the spectrum has a low-pass shape and that the components fall gradually. Disregarding the frequency dependency, a spectrum falling with a $2^{-k}$ decay (with $k$ taken as harmonic number) can be predicted. However, given that there is a substantial dependency on the fundamental, the spectral decay will be less accented. Figure 2 shows also that the FBAM waveform contains a significant DC component. By expanding (6) further, the static component is observed to be generated by the oddorder products of the summation.

Given the complexity of the product in (4), there is little more to be gained, as far as the spectral description of the sound is concerned, proceeding this way. We will instead turn to an alternative description of the problem, studying it as an IIR system.

2.2. Filter Interpretation. The FBAM algorithm can be interpreted as a coefficient-modulated one-pole IIR filter that is fed with a sinusoid. Rewriting (3) as

$$
y(n)=x(n)+a(n) y(n-1)
$$

with

$$
x(n)=a(n)=\cos \left(\omega_{0} n\right)
$$

results in a filter description for the algorithm, a periodically linear time-varying (PLTV) filter. This is a different system from the usual linear time-invariant (LTI) filters with static coefficients. Firstly, instead of a single fixed impulse response, this system has a periodically time-varying impulse response. Secondly, the filter's spectral properties are on their own functions of the discrete time: at each time sample, the filter transforms the input into an output signal depending on the coefficient values at that and preceding time instants. These types of filters were thoroughly investigated in $[6,7]$. Equation (11) of [6] defines a nonrecursive PLTV filter as

$$
y(n)=\sum_{k=0}^{N} b_{k}(n) x(n-k)
$$

The time-varying impulse response of a PLTV filter is defined in [6] as the output $y(n)$ measured at time $n$ in response to a discrete-time impulse $x(m)=\delta(m)$ applied at time $m$, and is given for the PLTV filter of (11) by (Equation (12) of [6])

$$
h(m, n)=\sum_{k=0}^{N} b_{k}(n) \delta(n-k-m) .
$$

Consequently, the filter's generalized transfer function (GTF) and generalized frequency response (GFR) $[6,7]$, which are the generalizations of the transfer function and frequency responses to the time-varying case, can be represented, respectively, as (Equations (2.14), (4.4), and (4.5) of [7])

$$
\begin{gathered}
H(z, n)=\sum_{m=-\infty}^{\infty} \sum_{k=0}^{N} b_{k}(n) \delta(n-k-m) z^{m-n}=\sum_{k=0}^{N} b_{k}(n) z^{-k}, \\
H(\omega, n)=\sum_{k=0}^{N} b_{k}(n) e^{-j k \omega} .
\end{gathered}
$$

The case of recursive PLTV filters, such as the one represented by FBAM, is more involved. The time-varying impulse response for the first-order recursive PLTV of (9) is given in [7] as

$$
h(m, n)= \begin{cases}\prod_{i=m+1}^{n} a(i)=\frac{g(n)}{g(m)}, & m<n, \\ 1, & m=n, \\ 0, & m>n, \\ 0, & n<0,\end{cases}
$$

with

$$
g(n)=\prod_{i=1}^{n} a(i) \quad \text { for } n \geq 1, g(0)=1 .
$$

The GTF of this filter is then defined as

$$
H(z, n)=\frac{\sum_{k=0}^{N-1} h(n-k, n) z^{-k}}{1-g(N) z^{-N}},
$$

where $N$ is the period in samples of the modulator signal $a(n)$. With this in hand, the time-varying frequency response of the filter in (9) can now be written as

$$
H(\omega, n)=\frac{\sum_{k=0}^{N-1} h(n-k, n) e^{-j k \omega}}{1-g(N) e^{-j N \omega}} .
$$


In the specific case of FBAM, (10) tells that the modulator signal $a(n)$ is a cosine wave with frequency $\omega_{0}=2 \pi f_{0} / f_{s}$ and period in samples $T_{0}=2 \pi / \omega_{0}$. In this case, to calculate the GTF for this filter, we can set $N=\left\lfloor T_{0}+0.5\right\rfloor$, where $\lfloor\cdot\rfloor$ is the floor function. Then, (17), (14), and (15) yield

$$
H(\omega, n)=\frac{1+\sum_{k=1}^{N-1} b_{k}(n) e^{-j k \omega}}{1-a_{N} e^{-j N \omega}},
$$

with the coefficients $b_{k}$ and $a_{N}$ set to

$$
\begin{gathered}
b_{k}(n)=\prod_{m=1}^{k} \cos \left(\omega_{0}[n-m+1]\right), \\
a_{N}=\prod_{m=1}^{N} \cos \left(\omega_{0} m\right) .
\end{gathered}
$$

The filter defined by (9) and (10) is therefore equivalent to a filter of length $N$, made up of a cascade of a time-varying FIR filter of order $N-1$ and coefficients $b_{k}(n)$, and an IIR (comb) filter with a fixed coefficient $a_{N}$. The equivalent filter equation is, thus,

$$
y(n)=x(n)+\sum_{k=1}^{N-1} b_{k}(n) x(n-k)+a_{N} y(n-N) .
$$

The recursive section does not have a significant effect on the FBAM signal, as the magnitude response peaks will line up with the harmonics of the fundamental. It will, however, have implications for the stability of the filter as will be seen later. The time-varying FIR section of this equivalent filter is then responsible for the generation of harmonic partials and the overall spectral envelope of the signal. In [7], these partials are called combinational components, which are added to the output in addition to the input signal spectral components (which in the case of FBAM are limited to a single sinusoid). Plots of the output of this filter when fed with a sinusoid with radian frequency $\omega_{0}=2 \pi f_{0} / f_{s}$ and its equivalent FBAM signal are shown in Figure 3.

Studies have shown that modulation of IIR filter coefficients (such as the coefficient-modulated allpass) has a phase-distortion effect on the input signal [8-10]. In addition, the amplitude modulation effect caused by the time-varying magnitude response will help in shaping the output signal. To demonstrate this, the FBAM signal can be reconstituted using phase and amplitude modulation, defined by

$$
y(n)=A(n) \cos \left(\omega_{0} n+\phi(n)\right),
$$

where

$$
A(n)=\left|H\left(\omega_{0}, n\right)\right|, \quad \phi(n)=\arg \left(H\left(\omega_{0}, n\right)\right),
$$

with $H(\omega, n)$ defined by (18) and setting $\omega=\omega_{0}$. A plot of this reconstruction and its equivalent FBAM waveform is shown on Figure 4, where the steady-state signals are seen to match each other. It is worth pointing out that this result can be alternatively inferred from the similarities between the periodic time-varying filter transfer function and the expansion of the FBAM expression in (4).

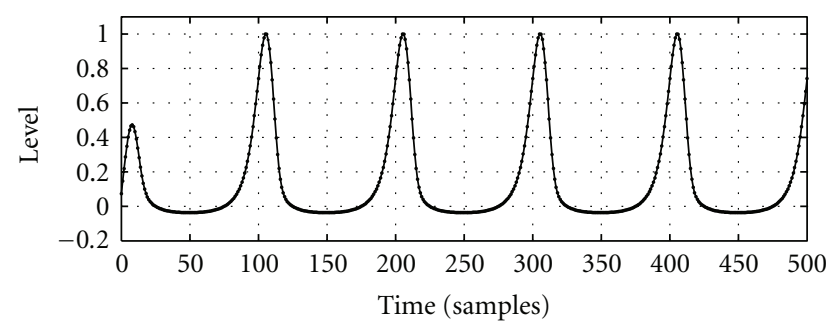

Figure 3: Plots of the FBAM waveform (dots) and the output of its equivalent time-varying filter of (20) (solid), when fed with a sinusoid $\left(f_{0}=441 \mathrm{~Hz}\right)$.

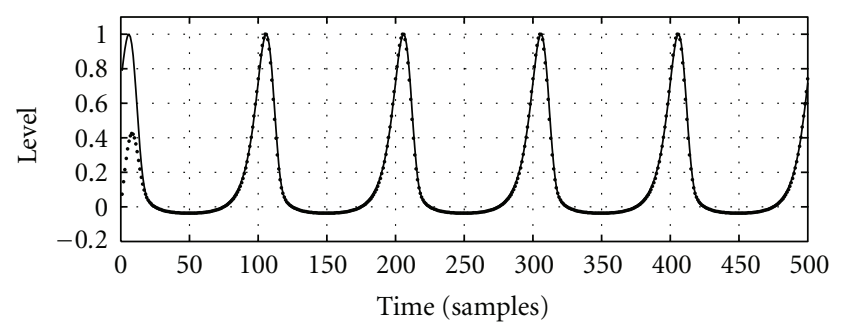

FIGURE 4: Plot of the reconstructed FBAM signal (solid) against the actual FBAM waveform (dots), with $f_{0}=441 \mathrm{~Hz}$. The reconstruction is based on the steady-state spectrum and thus does not include the transient effect seen at the start of the FBAM waveform.

2.3. The Basic FBAM Equation. To make the algorithm more flexible, some means of controlling the amount of modulation (and therefore, distortion) is inserted into the system. This can be effected by introducing a modulation index $\beta$ into (3), which yields

$$
y(n)=\cos \left(\omega_{0} n\right)[1+\beta y(n-1)]
$$

The flowchart of this equation is shown in Figure 5. By varying the parameter $\beta$, it is possible to produce dynamic spectra, from a pure sinusoid to a fully-modulated signal with various harmonics. The action of this parameter is demonstrated in Figure 6, which shows the spectrogram of a FBAM signal with $\beta$ sweeping linearly from 0 to 1.5 . The signal bandwidth and the amplitude of each partial increase with the $\beta$ parameter. Notice that this is a simpler relation than in frequency modulation (FM) synthesis [11], in which partials are momentarily faded out as the modulation index is changed (see, e.g., Figure 4.2 on page 301 in [12]).

The maximum value of $\beta$ will mostly depend on the tolerable aliasing levels, as higher values of $\beta$ will increase the signal bandwidth significantly. Even higher values of this parameter will also cause stability problems, which are discussed below.

2.4. Stability and Aliasing. The stability of time-varying filters is generally difficult to guarantee [13]. However, in the present case, it is possible to have a stable algorithm by controlling the amount of feedback in the system. From (20) 


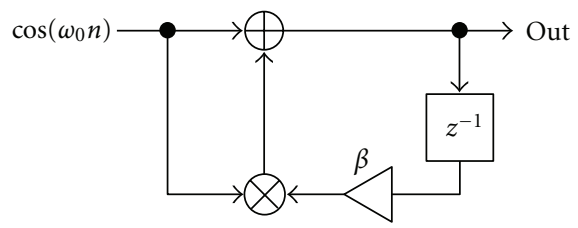

FIGURE 5: Flowchart of the basic FBAM equation, where $z^{-1}$ denotes the delay of a unit sample period.

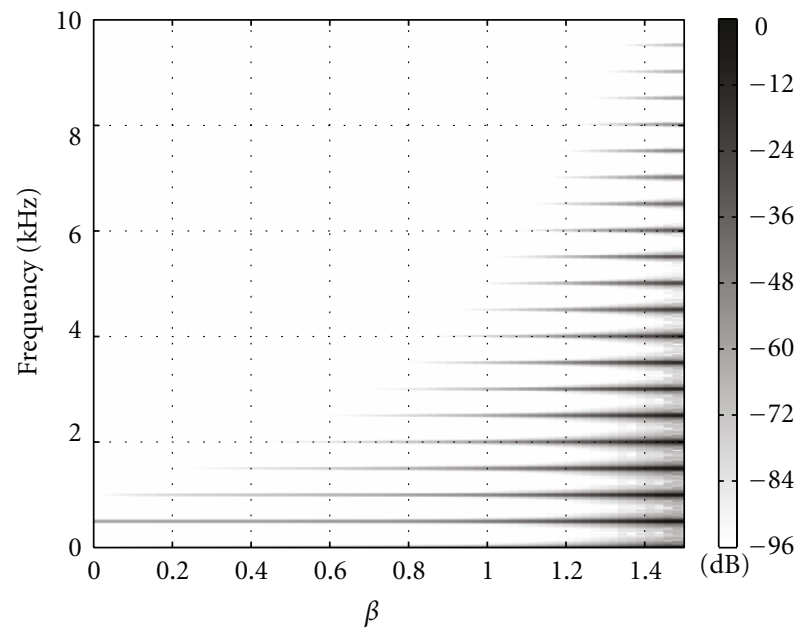

FIGURE 6: Spectrogram of the FBAM output with $\beta$ varying from 0 to $1.5\left(f_{0}=500 \mathrm{~Hz}\right)$.

and (23), the impulse response of the system is noted to decrease in time when

$$
\left|\beta a_{N}\right|<1
$$

that is, when the product of instantaneous coefficient values over the period multiplied by the modulation index $\beta$ is less than unity [7]. The dashed line of Figure 7 plots the maximum $\beta$ values satisfying this stability condition, showing that the stability is frequency dependent. The approximate stability limit is given by $\beta_{\text {stable }} \approx 1.9986-0$. $00003532\left(f_{0}-27.5\right)$

In practice, however, the system stability will never become the limiting issue. This is because for values of $\beta$ well within the range of stable values, an objectionable amount of aliasing is obtained. So, in fact, the real question is how large can the modulation index be before the digital baseband is exceeded. This will of course depend on the combination of the sampling rate and fundamental frequency. Taking for instance $f_{0}=500 \mathrm{~Hz}$ and $f_{s}=44100 \mathrm{~Hz}$, one observes that for $\beta=1.9$, there is considerable foldover distortion throughout the spectrum (see Figure 8). The distortion is also visible in the signal waveform as the formation of wave packets similar to those found in overmodulated feedback FM synthesis [14].

The solid and dotted curves in Figure 7 show the maximum $\beta$ values that keep the amount of aliasing $80 \mathrm{~dB}$ below the loudest harmonic (the fundamental) at sample rates of $44.1 \mathrm{kHz}$ and $88.2 \mathrm{kHz}$, respectively. The curves were

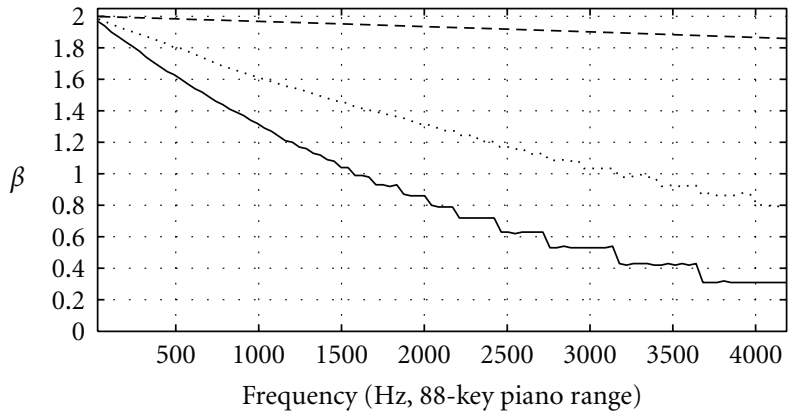

FIGURE 7: Stability (dashed) and aliasing (solid: $f_{s}=44.1 \mathrm{kHz}$, dotted: $f_{s}=88.2 \mathrm{kHz}$ ) limits of FBAM.

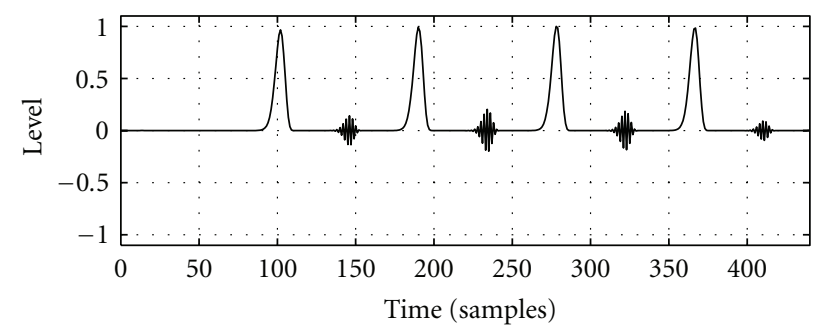

(a)

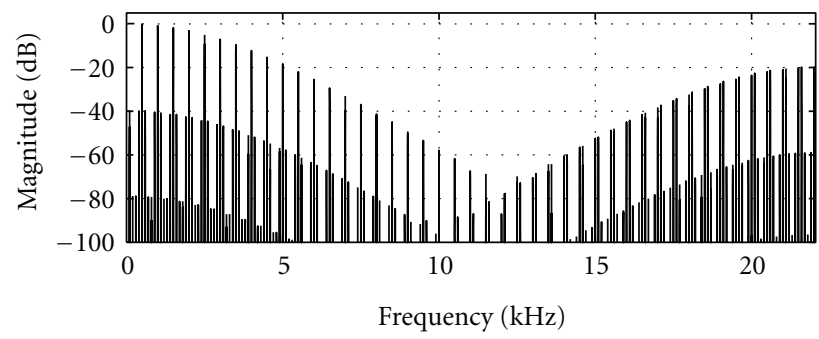

(b)

Figure 8: FBAM spectrum and waveform with $\beta=1.9\left(f_{0}=\right.$ $500 \mathrm{~Hz})$.

obtained through iterated spectral analysis: the frequency axis was sampled at 100 points, and for each fundamental frequency, the $\beta$ value was increased until the magnitude of the strongest aliasing harmonic reached the $-80 \mathrm{~dB}$ limit (the algorithm is available at [15]). The solid curve $\left(f_{s}=\right.$ $44.1 \mathrm{kHz}$ ) shows that for fundamental frequencies lower than $1300 \mathrm{~Hz}$, when the curve is smooth, the maximum usable $\beta$ values are determined by the overmodulation foldover distortion discussed above. For higher fundamental frequencies, the stepwise shape of the curve suggests that the $-80 \mathrm{~dB}$ limit is determined by the harmonics folding back to the digital baseband at the Nyquist limit. The dotted curve $\left(f_{s}=88.2 \mathrm{kHz}\right)$ shows that oversampling increases the usable $\beta$ range by stretching the maximum $\beta$ values towards higher frequencies relative to the oversampling amount.

2.5. Scaling. The gain of the FBAM system varies considerably with different $\beta$ values - in a frequency-dependent manner-and grows rapidly after $\beta$ exceeds unity. This makes the output gain normalization a challenge, which 


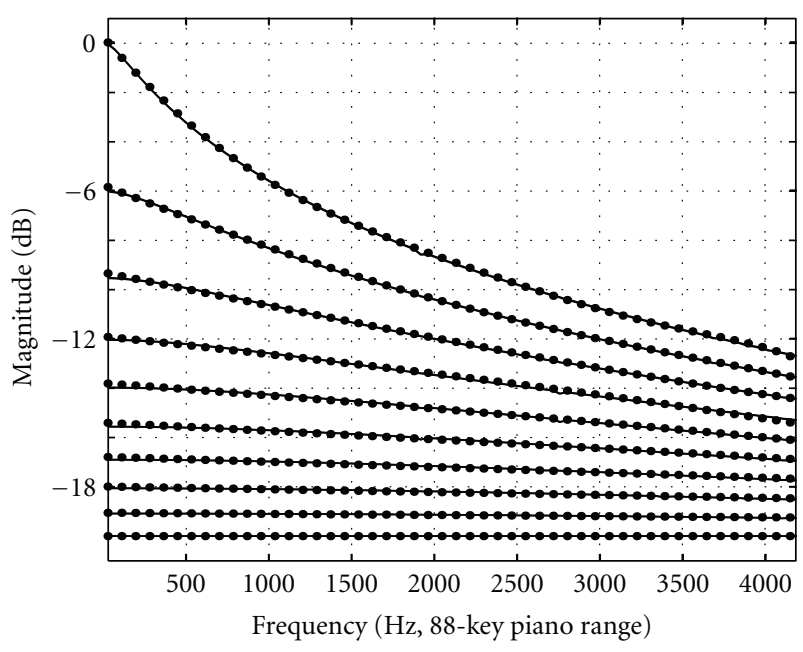

FIGURE 9: FBAM gain (solid) and its polynomial approximation (dotted). $\beta=0.1$ (bottom) $\cdots \beta=0.9$ (top).

can, however, be resolved by approximate peak-scaling and average power balancing algorithms.

Figure 9 shows that the peak gain of the basic FBAM equation (solid line) can be approximated well within a 1$\mathrm{dB}$ deviation by polynomials of degree $1(\beta<0.7)$ and of degrees 2,3 , and 5 (corresponding to $\beta$ values $0.7,0.8$, and 0.9 , resp.).

The scaling factors for in-between $\beta$ values can be found by linear interpolation, provided that the polynomial approximations are taken at sufficiently small intervals (e.g., setting $\Delta \beta=0.05$ generated acceptable results). Scaling factors for $\beta \geq 1$ follow power-law approximations, which are problematic with low fundamental frequencies where the FBAM gain rate changes most rapidly. A two-dimensional lookup table $(\Delta \beta=0.05,100$ frequency samples) with bilinear interpolation was found to be able to provide more accurate results across the entire stable $\beta$ range. Each entry in the table can be precalculated by evaluating one half period of (23) using a sine input and finding the maximum value of the result. The lookup table and the function coefficients are available at [15]. The two-dimensional lookup table approach was observed to provide transient-free scaling for control rate parameter sweeps.

Equation (23) may alternatively be evaluated at the control rate for each block of output samples. Another online solution is to use a root-mean-square (RMS) balancer [1] that consists of two RMS estimators and an adaptive gain control. The FBAM output and cosine comparator signals are first fed into the RMS estimators, which rectify and low-pass filter their inputs to obtain the estimates. The scaling factor is then calculated from a ratio of the two RMS estimates. This solution is sufficiently general to work with the variations discussed in the next section.

\section{Variations}

The basic structure of FBAM provides an interesting platform on which new variants can be constructed. This section will examine a number of these (see Figure 10), starting from the insertion of a feedforward term, which can subsequently be used for an allpass filter-derived structure, and proceeding to heterodyning, nonlinear distortion, nonunitary delays, and the generalization of FBAM as a coefficient-modulated filter.

3.1. Variation 1: Feedforward Delay. A simple way of generating a different waveshape is to include a feedforward delay term in the basic FBAM equation (see Figure 10(a))

$$
y(n)=\cos \left[\omega_{0}(n-1)\right]-\cos \left(\omega_{0} n\right)[1+\beta y(n-1)] .
$$

In this case, besides the DC offset, there is no change in the spectrum as the feedforward delay will not change the shape of the input (i.e., it remains a sinusoid). However, because of the half-sample delay caused by the feedforward section, the shape of the waveform is different, as its harmonics are given different phase offsets. Figure 11 shows the waveform and spectrum of this FBAM variant.

3.2. Variation 2: Coefficient-Modulated Allpass Filter. From the feedforward delay variation discussed above, it is possible to derive a variant that is similar to the coefficient-modulated allpass filter described in [8] and used for phase distortion synthesis in $[9,10]$. The general form of this filter is

$$
y(n)=x(n-1)-a(n)[x(n)-y(n-1)] .
$$

This is translated into the presented FBAM form by equating $a(n)$ to the input signal $x(n)=\cos \left(\omega_{0} n\right)$, as in Section 2.1

$$
y(n)=\cos \left[\omega_{0}(n-1)\right]-\beta \cos \left(\omega_{0} n\right)\left[\cos \left(\omega_{0} n\right)-y(n-1)\right] .
$$

The flowchart of the coefficient-modulated allpass filter is shown in Figure 10(b), while its waveform and spectrum are plotted in Figure 12.

The resulting process is equivalent to a form of phase modulation synthesis, as discussed in [9]. As with the basic version of FBAM, it is possible to raise the modulation index $\beta$ above one, as this variant exhibits similar stability and aliasing behavior.

3.3. Variation 3: Heterodyning. Employing a second sinusoidal oscillator as a ring-modulator provides a further variant to the basic FBAM method. This heterodyning variant can have two forms, by placing the modulator inside or outside the feedback loop, as shown in Figures 10(c) and $10(d)$, respectively, producing different output spectra.

3.3.1. Type I: Modulator inside the Feedback Loop. In this structure, the basic FBAM expression is simply multiplied by a cosine wave of a different frequency

$$
y(n)=\cos (\theta n)\left\{\cos \left(\omega_{0} n\right)[1+\beta y(n-1)]\right\},
$$

where $\theta$ is the normalized radian frequency of the ringmodulator. The main characteristic of this variant is that the 


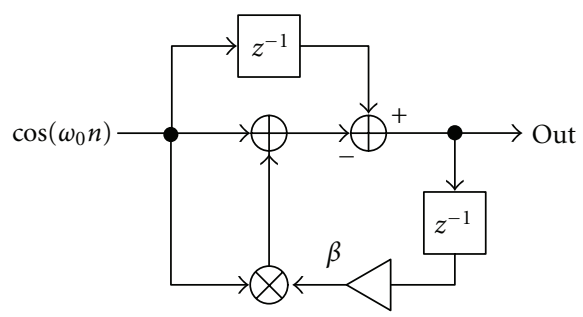

(a)

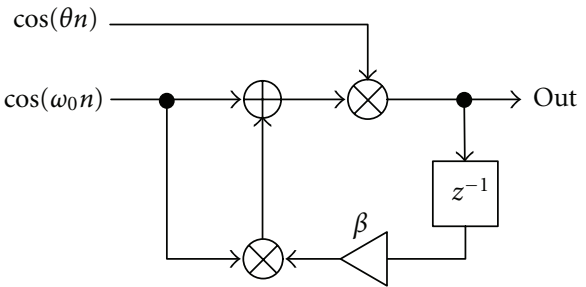

(c)

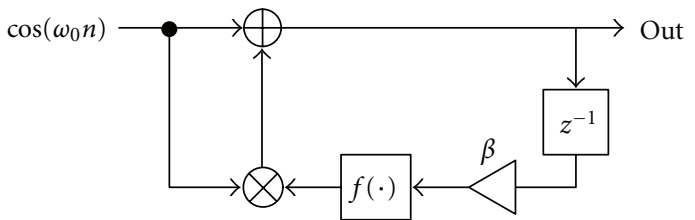

(e)

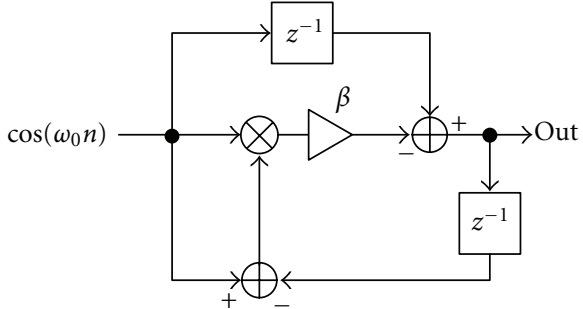

(b)

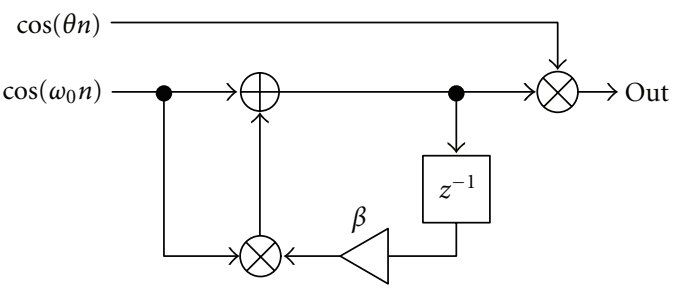

(d)

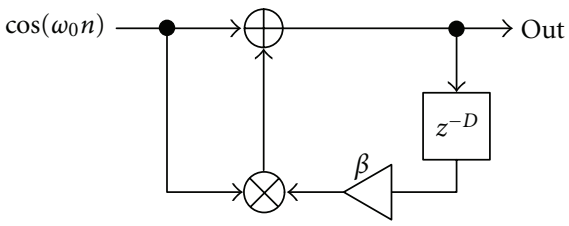

(f)

FIgURE 10: FBAM variation flowcharts. The $z^{-1}$ and $z^{-D}$ symbols denote delays of one and $D$ sample periods, respectively.

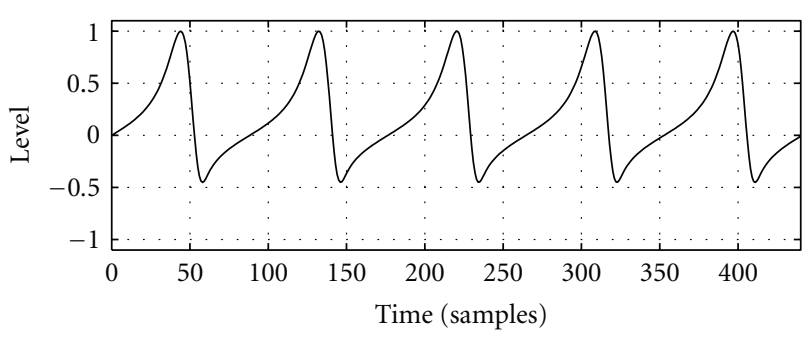

(a)

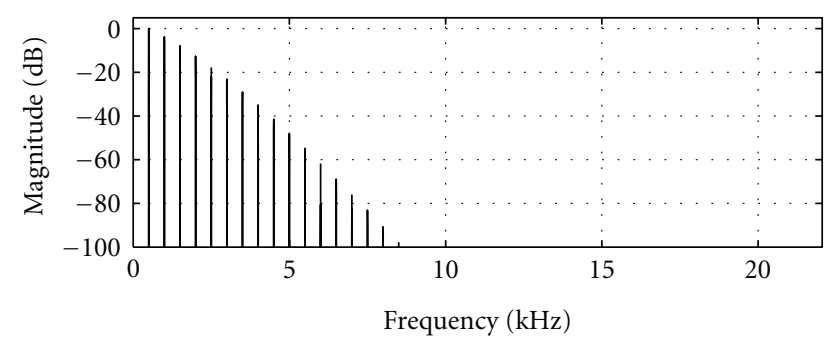

(b)

FIGURE 11: Waveform and spectrum of FBAM variation $1(\beta=1$, $\left.f_{0}=500 \mathrm{~Hz}\right)$, see Figure 10(a).

whole of the modulated signal is fed back to modulate the amplitude of the first oscillator, as shown in Figure 10(c). In general, if the ratio of frequencies of the modulator and FBAM oscillators is of small integers, the result is a harmonic

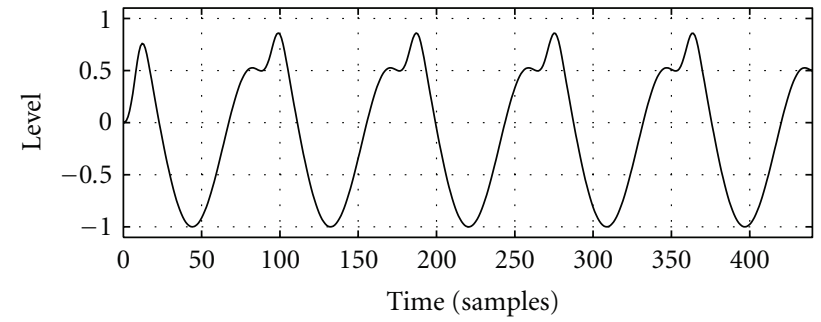

(a)

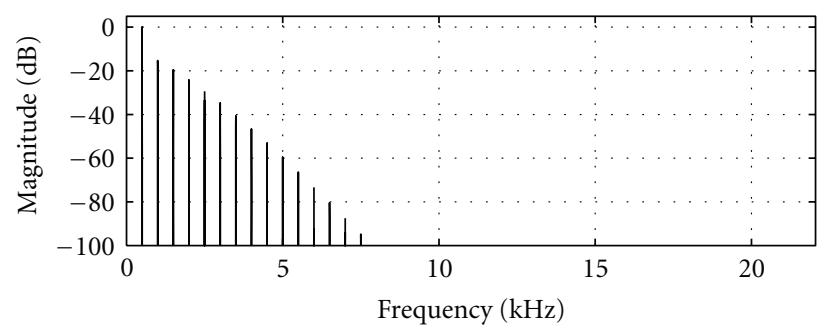

(b)

Figure 12: Waveform and spectrum of FBAM variation $2(\beta=1$, $f_{0}=500 \mathrm{~Hz}$ ), see Figure 10(b).

spectrum. This ratio also determines the general shape of the spectrum, which exhibits regularly-spaced peaks. Both the fundamental frequency and the spacing of peaks are dependent on this frequency ratio. 


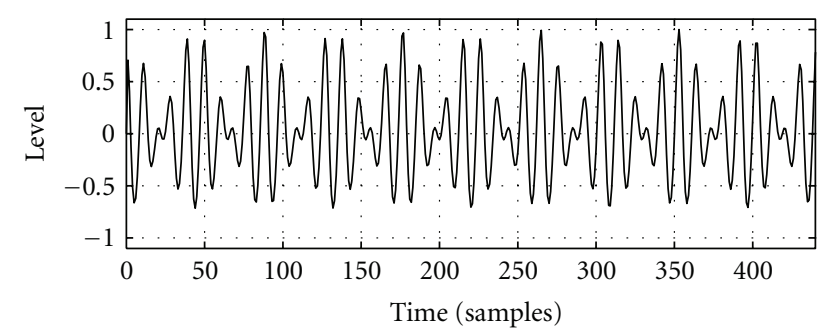

(a)

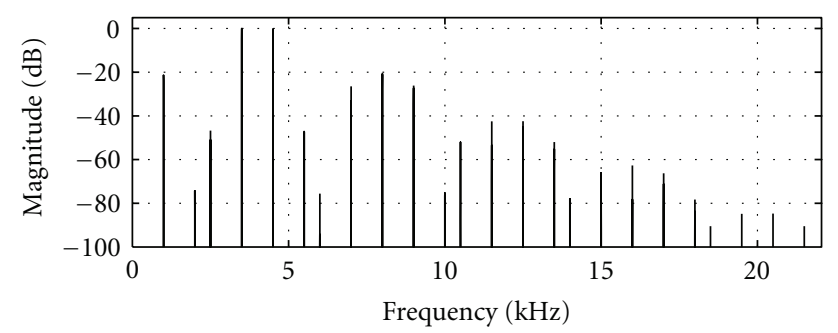

(b)

Figure 13: Heterodyne FBAM variation 3 -I $\left(f_{0}=500 \mathrm{~Hz}, \beta=0.2\right.$, modulator frequency $4000 \mathrm{~Hz}$ ( $8: 1$ ratio)), see Figure 10(c).

In some cases, harmonics are missing or they have very small amplitudes, such as in the case of the $8: 1$ ratio shown in Figure 13. Here, harmonics 1, 3, 6, 8, 10, 13, 15, 17, 19, $22,24,26$, and so forth are seen to be missing (or have an amplitude at least $-100 \mathrm{~dB}$ from the maximum). The peaks in the spectrum are around harmonics 8 (missing), 16, 24 (missing), 32 and 40 (missing). This method provides a rich source of spectra. However, its mathematical description is very complex and the matching of parameters to the spectrum is not as straightforward as in other variants. On the plus side, the $\beta$ parameter (FBAM modulation index) maps simply to spectral richness and it does not have a major effect on the relative amplitude of harmonics (beyond that of adding more energy to higher components). However, because of aliasing issues, the practical $\beta$ range decreases rapidly with increasing $\theta / \omega_{0}$ ratios.

3.3.2. Type II: Modulator outside the Feedback Loop. The second form of heterodyne FBAM places the modulation outside the feedback loop (see Figure 10(d)). In other words, the basic FBAM algorithm is used to create a modulator signal with a baseband spectrum, which is then shifted to be centered on the cosine carrier frequency $\theta$, as defined by the following pair of equations:

$$
\begin{gathered}
y(n)=\cos \left(\omega_{0} n\right)[1+\beta y(n-1)], \\
s(n)=\cos (\theta n) y(n) .
\end{gathered}
$$

A similar structure is seen in the double-sided Discrete Summation Formula (DSF) algorithm [16], as well as in PhaseAligned Formant (PAF) synthesis [17] (which is derived from DSF) and phase-synchronous Modified FM [18, 19]. This heterodyne principle is very useful for generating resonant spectra and formants by setting $\theta=k \omega_{0}$, with $k>0$ and an integer, that is, making the cosine frequency a

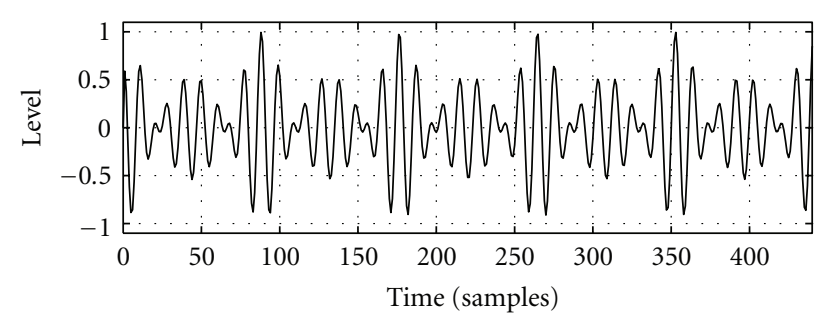

(a)

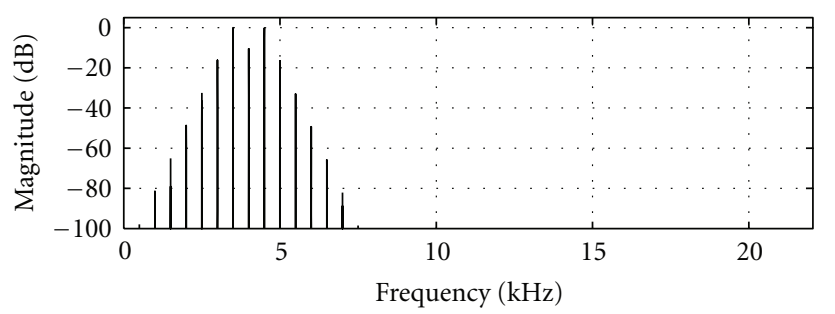

(b)

Figure 14: Heterodyne FBAM variation 3 -II $\left(f_{0}=500 \mathrm{~Hz}, \beta=0.3\right.$, cosine carrier frequency $4000 \mathrm{~Hz}$ ( $8: 1$ ratio)), see Figure 10(d).

multiple of the FBAM $f_{0}$. Figure 14 depicts the waveform and spectrum of (29), with $k=8\left(\beta=0.3, f_{0}=500 \mathrm{~Hz}\right.$, and $f_{s}=44100 \mathrm{~Hz}$ ). Note that the bandwidth of the resonant region is proportional to $\beta$ and that the practical $\beta$ range is considerably wider than in heterodyning type I.

A more general algorithm for formant synthesis would require the use of two carriers tuned to adjacent harmonics around the resonance frequency $f_{\mathrm{c}}$, whose signals are weighted and mixed together to provide the output

$$
\begin{gathered}
k=\operatorname{int}\left(\frac{f_{\mathrm{c}}}{f_{0}}\right), \\
g=\frac{f_{\mathrm{c}}}{f_{0}}-k \\
y(n)=\cos \left(\omega_{0} n\right)[1+\beta y(n-1)], \\
s(n)=y(n)\left\{(1-g) \cos \left(k \omega_{0} n\right)+g \cos \left[(k+1) \omega_{0} n\right]\right\} .
\end{gathered}
$$

This structure can be used for efficient synthesis of resonances from vocal formants to emulation of analogue synthesizer sounds.

3.4. Variation 4: Nonlinear Waveshaping. An interesting modification of the FBAM algorithm can be implemented by employing a nonlinear mapping of the feedback path, a process commonly known as waveshaping [20, 21]. The general form of the algorithm is

$$
y(n)=\cos \left(\omega_{0} n\right)\{1+f[\beta y(n-1)]\},
$$

where $f(\cdot)$ is an arbitrary nonlinear waveshaper (Figure 10(e)). There are a variety of possible transfer functions that may be employed for this purpose. The most useful ones appear to be trigonometric $(\sin (\cdot), \cos (\cdot)$, etc.) 


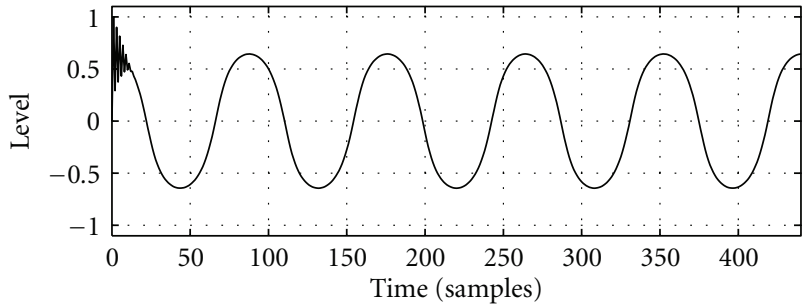

(a)

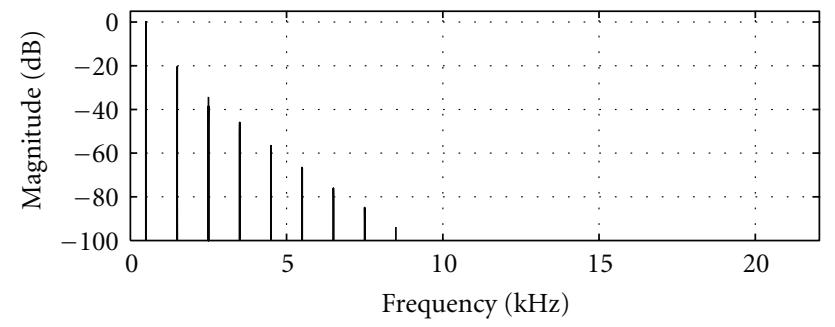

(b)

FIGURE 15: FBAM variation 4 with cosine waveshaping $\left(\beta=1, f_{0}=\right.$ $500 \mathrm{~Hz}$ ), see Figure 10(e). The transient appears because the initial state of the filter was not set up appropriately.

and a few piecewise-linear waveshapers (such as the absolute value function ABS).

The case of cosine and sine waveshapers is particularly interesting; for instance,

$$
y(n)=\cos \left(\omega_{0} n\right)\{1+\cos [\beta y(n-1)]\}
$$

produces a signal that is closely related to feedback FM synthesis [22]. To demonstrate the similarities, start with the FM equation [11]

$$
y(n)=\cos \left[\omega_{0} n+m(n)\right]
$$

and set the modulator function $m(n)=y(n-1)$ to implement the feedback. Expanding this gives

$$
\begin{aligned}
y(n) & =\cos \left[\omega_{0} n+y(n-1)\right] \\
& =\cos \left(\omega_{0} n\right) \cos [y(n-1)]-\sin \left(\omega_{0} n\right) \sin [y(n-1)] .
\end{aligned}
$$

So, the cosine-waveshaped FBAM partially implements the feedback FM equation. As it turns out, this partial implementation removes all even harmonics from the spectrum. This is shown in Figure 15, which illustrates also the effect of an improper initial state: the waveform contains a transient, which is due to a poorly chosen initial feedback state value. Here, $y(0)=1$ instead of the recommended peak value of the steady-state waveform.

It is possible to closely approximate feedback FM by combining two sinusoidal waveshaper FBAM structures, one of them using cosine and the other sine functions

$$
\begin{aligned}
y(n)= & \cos \left(\omega_{0} n\right)\{1+\cos [\beta y(n-1)]\} \\
& -\sin \left(\omega_{0} n\right)\{1+\sin [\beta y(n-1)]\} \\
= & \cos \left[\omega_{0} n+\beta y(n-1)\right]+\cos \left(\omega_{0} n\right)-\sin \left(\omega_{0} n\right) .
\end{aligned}
$$

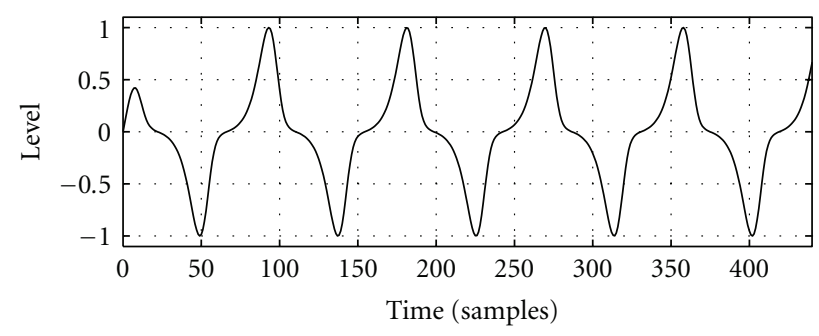

(a)

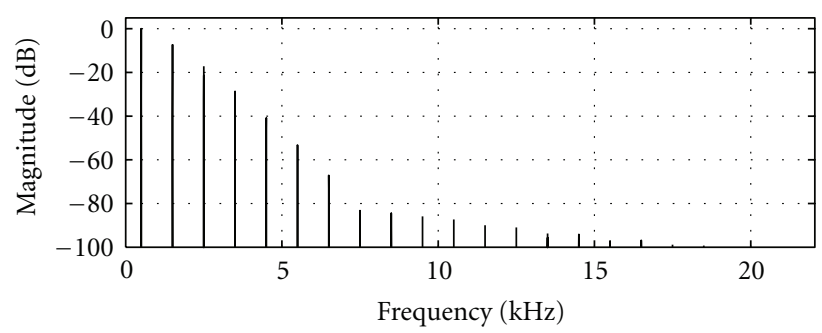

(b)

FIGURE 16: FBAM variation 4 with ABS waveshaping $\left(\beta=1, f_{0}=\right.$ $500 \mathrm{~Hz}$ ), see Figure 10(e).

As can be seen, this expression only differs from feedback FM by the added sine and cosine components at $f_{0}$. Equation (37) demonstrates that it is possible to create transitions between cosine (and sine) waveshaped FBAM and feedback FM. This might be a useful feature to be noted in implementations of the technique.

Choosing the ABS transfer function provides another means of removing even harmonics from the FBAM spectrum, as shown in Figure 16. This is because, like the cosine waveshaper, the absolute value function is an even function. Such a waveshaper will feature only even harmonics of its input signal frequencies [19]. However, in the current setup, the waveshaper output is heterodyned by a cosine wave tuned to its fundamental frequency, thus generating odd harmonics of that frequency.

Another interesting feature of the ABS waveshaper is that it maintains the relative amplitudes of odd components close to the values in the basic FBAM expression. Therefore, it provides an interesting means of varying odd-even balance of a synthesized tone by combining this variant with the basic FBAM technique.

The aliasing properties of variation 4 depend naturally on the choice of the waveshaper. For the presented cases, the practical $\beta$ range is slightly more restricting than the general case shown in Figure 7.

3.5. Variation 5: Nonunitary Feedback Periods. The early works on feedback amplitude modulation utilized various feedback delay lengths. In [3], Risset does not discuss the design in detail, but from his MUSIC V code the feedback delay is seen to be one sample block (existing FORTRAN code shows that the program processes the signal on a block-by-block basis [23]). Layzer's article [4] describes the algorithm as based on a fixed feedback delay of 512 samples (the system block size). A footnote mentions an alternative 
implementation by F.R. Moore allowing delays from one to 512 samples. In [5], the feedback delay is equivalent to the default processing block size for the system in which it is implemented (64 samples). The differences in feedback delay lengths are important to the resulting output.

The feedback delay of the basic FBAM can be generalized to allow for an arbitrary period size (see Figure 10(f)). Instead of limiting the delay to one sample, it can be made variable

$$
y(n)=\cos \left(\omega_{0} n\right)[1+\beta y(n-D)]
$$

where $D$ is the delay length in samples. From a filter perspective, this equation defines a coefficient-modulated comb filter (which is fed a cosine wave as input). As such the delay $D$ can be expected to have an effect on the output spectrum. Different waveshapes can be produced with various delays, but the spectrum will be invariant if the ratio of the delay time $T_{D}=f_{s} / D$ and the modulation frequency, which is in this case also $f_{0}$, is preserved. For this to be effective, the delay time will be inversely proportional to the change in fundamental frequency. This principle should additionally allow keeping the basic FBAM spectrum $f_{0}$ invariant by lengthening the delay as frequency decreases. Of course, there will be an upward limit of one-sample delay (if fractional delays are not desired).

An interesting case arises when the $T_{D}: f_{0}$ ratio is one, and so $D=2 \pi \omega_{0}^{-1}=f_{s} / f_{0}$. In this case, the FBAM expression becomes much simpler

$$
\begin{aligned}
y(n) & =\cos \left(\omega_{0} n\right)\left[1+\beta y\left(n-\frac{2 \pi}{\omega_{0}}\right)\right] \\
& =\sum_{k=0}^{\infty} \beta^{k} \prod_{m=0}^{k} \cos \left(\omega_{0} n-2 \pi m\right) \\
& =\sum_{k=1}^{\infty} \beta^{k-1} \cos \left(\omega_{0} n\right)^{k}=\frac{\cos \left(\omega_{0} n\right)}{1-\beta \cos \left(\omega_{0} n\right)},
\end{aligned}
$$

for $0 \leq \beta<1$ (see Figure 17); with $\beta=1$, there is a singularity at $\cos (0)$, and with $\beta>1$, the series is divergent and the closed form does not apply. It is also possible to expand the summation in (39) to obtain its spectra

$$
\begin{aligned}
& \sum_{k=1}^{\infty} \beta^{k-1} \cos \left(\omega_{0} n\right)^{k} \\
& =\sum_{k=1}^{\infty} \beta^{2 k-1} \cos \left(\omega_{0} n\right)^{2 k}+\beta^{2 k-2} \cos \left(\omega_{0} n\right)^{2 k-1} \\
& =\sum_{k=1}^{\infty} \beta^{2 k-1}\left\{\frac{1}{2^{2 k}}\left(\begin{array}{c}
2 k \\
m
\end{array}\right)+\frac{2}{2^{2 k}} \sum_{m=0}^{k-1}\left(\begin{array}{c}
2 k \\
m
\end{array}\right) \cos \left[(2 k-2 m) \omega_{0} n\right]\right\} \\
& +\frac{2 \beta^{2 k-2}}{2^{2 k-1}} \sum_{m=0}^{k-1}\left(\begin{array}{c}
2 k-1 \\
m
\end{array}\right) \cos \left[(2 k-2 m-1) \omega_{0} n\right] .
\end{aligned}
$$

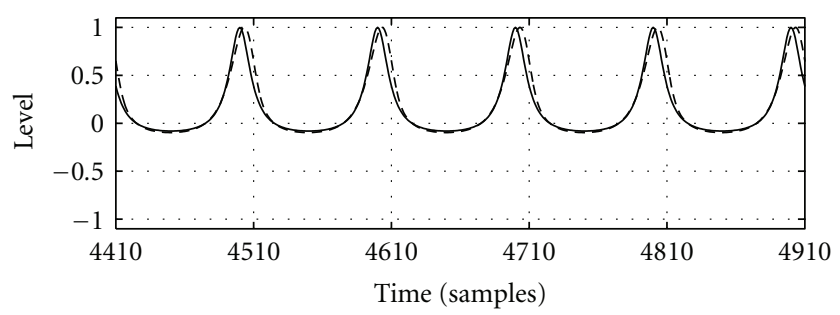

(a)

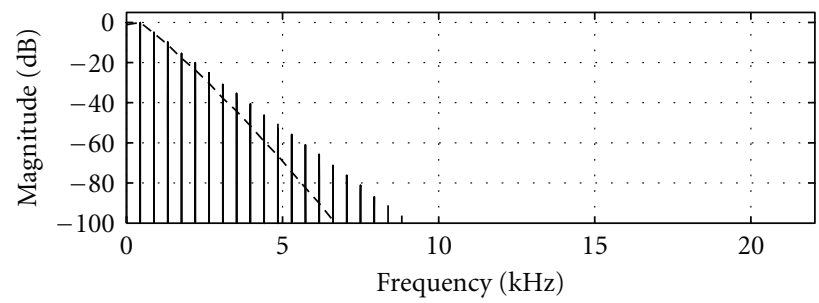

(b)

FiguRE 17: FBAM variation $5\left(\beta=0.85, f_{0}=441 \mathrm{~Hz}\right)$ with feedback period $D=100$ (solid), see Figure 10(f). The dashed line plots the basic FBAM with period $D=1$.

To gain an understanding of the type of spectra obtained, (40) can be partially evaluated limiting $k$ to 4

$$
\begin{aligned}
& \sum_{k=1}^{4} \beta^{k-1} \cos \left(\omega_{0} n\right)^{k} \\
& =\frac{1}{2} \beta+\frac{3}{8} \beta^{3}+\left[1+\frac{3}{4} \beta^{2}\right] \cos \left(\omega_{0} n\right)+\left[\frac{\beta-\beta^{3}}{2}\right] \cos \left(2 \omega_{0} n\right) \\
& \quad+\frac{1}{4} \beta^{2} \cos \left(3 \omega_{0} n\right)+\frac{1}{8} \beta^{3} \cos \left(4 \omega_{0} n\right) .
\end{aligned}
$$

In order to obtain a continuous range of delay times, some form of interpolation is required. As observed in [24], this will have an effect on the output. Although it is beyond the scope of the present study to discuss the best interpolation methods for fractional delay FBAM, good results have been observed with a linear interpolation method in delays longer than a few samples. For very short delays, a higher precision interpolator would most likely be required.

The aliasing properties of variation 5 follow closely the general case of Figure 7. However, we observed that the system becomes unstable with large $\beta$ values when $D=f_{s} / f_{0}$ or $D=f_{s} / 2 f_{0}$.

3.6. Variation 6: Coefficient-Modulated First-Order IIR Filter. So far, the focus has been on self-modulation scenarios that share a single sinusoid between the carrier and the modulating signal. The FBAM algorithm is now generalized as a coefficient-modulated IIR filter by relaxing the constraint of (10) and decoupling the input signals, that is, the carrier $x(n)$ and the modulator $m(n)$, into independent and arbitrary inputs as shown in Figure 18. Rephrasing (23) as

$$
y(n)=x(n)+m(n) \beta y(n-1)
$$




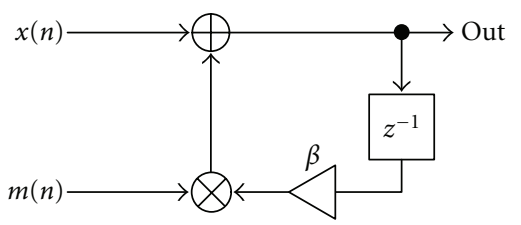

FigURE 18: FBAM variation 6 with decoupled input signals.

offers two additional degrees of freedom, because the frequencies and the waveforms of both input signals can now be chosen independently. In the present study, the modulator signal should be periodic, however.

For sinusoidal carrier and modulator signals with $\beta=0$, the spectrum of (42) consists of a single component that is located at the carrier frequency $f_{x}$. As $\beta$ is increased, the component at $f_{x}$ is skirted with upper and lower sidebands and the resultant spectra will gradually grow into the formant-shaped structure shown in Figure 14. The spectral structure of the formant can be controlled by the modulation frequency $f_{m}$, as the sideband components will appear at frequencies $f_{x} \pm k f_{m}$. Negative frequencies alias at DC back to the positive domain, and, as discussed in Section 3.3, frequency ratios $f_{x}: f_{m}$ of small integers will generate harmonic spectra, while more complex ratios result in inharmonic timbres.

A complex periodic modulation signal generates upper and lower sidebands for each of its spectral component, which happens also in complex modulator FM $[25,26]$. The sidebands consist of partials that are located at frequencies $\pm k f_{x}$ around the original modulator component, and, therefore, the spectral structure of the formants can be controlled by the carrier oscillator frequency $f_{x}$.

The aliasing properties of variation 6 depend on the frequency ratio between the carrier and the modulator. Larger frequency ratios increase the amount of high end spectral content, and, therefore, the risk of aliasing is bigger than in the general case of $f_{x}: f_{m}=1$.

3.6.1. Adaptive Feedback AM. The filter interpretation of FBAM allows its treatment as an adaptive audio effect. This can be implemented in two general lines, based on how the input signal shall be used to control the filter behavior. The first and the simplest method is to exchange the sinusoidal signal with an arbitrary input. In this method, the filter coefficient will be modulated by the input

$$
y(n)=x(n)[1+\beta y(n-1)] .
$$

The second alternative implementation, based on (42) and following the principles adopted in Adaptive FM [27], uses a pitch tracking algorithm to control the modulator frequency. In addition, the value of $\beta$ can be made proportional to the amplitude of the input signal with the use of an envelope follower. This method is perhaps more flexible as one can avail of a choice of modulator signals and a different modulator frequency (whereas the first method locks a $1: 1$ ratio between the input and coefficient modulator). In addition, both alternatives can also avail of nonunitary delay
TABLE 1: FBAM input signals and synthesis parameters.

\begin{tabular}{lccc}
\hline Input & Category & Variation & Description \\
\hline$x(n)$ & Signal & Common & Carrier \\
$m(n)$ & Signal & Common & Modulator \\
$r(n)$ & Signal & 3 & Ring modulator \\
$v$ & Parameter & Common & Variation number \\
$\beta$ & Parameter & Common & Feedback amount \\
$w_{s}$ & Parameter & 4 & Waveshaper type \\
$D$ & Parameter & 5 & Feedback delay length \\
\hline
\end{tabular}

sizes, which would allow different spectral characteristics at the output (and of course, in this case, we are no more strictly speaking of a first-order filter structure).

The stability of the first method depends on the input signal matching the conditions set by (24). A special case might occur when the input signal is not periodic, in which case the PLTV filter theory does not apply. The second method follows the stability condition as studied in Section 2.4. The aliasing properties depend largely on the input waveform.

\section{Implementations}

This section refines the FBAM operator and algorithm concepts introduced in [2] and discusses their implementation in two popular audio synthesis environments. These concepts are related to their FM synthesis counterparts described in [28].

4.1. FBAM Operator. The audio rate interface of the FBAM flowcharts depicted in Figures 5 and 10 consists of two input $\left(\cos \left(\omega_{0} n\right), \cos (\theta n)\right)$, and one output (Out) signals. The shared $\cos \left(\omega_{0} n\right)$ input can be further decoupled into separate carrier and modulator signals $x(n)$ and $m(n)$, as discussed in Section 3.6. The control rate interface of the flowcharts consists of the synthesis parameters. By hiding the implementation details of the variations, the FBAM system can be considered as a black box that is interfaced with the inputs listed in Table 1. The contents of the FBAM black box includes a waveshaper (variation 4), a delay line (variation 5), an output level scaling unit, and a set of state variables, multipliers, and adders for the algorithmic details, as shown in the FBAM flowcharts.

Cascading a sinusoidal carrier oscillator, the FBAM black box, and an envelope-controlled gain unit brings forth the Feedback AM Operator shown in Figure 19. The signal inputs $r(n)$ and $m(n)$ can be derived from the outputs of other FBAM operators or from the carrier oscillator output $x(n)$ by means of the modulation source selector $S_{M}$.

To simplify the tuning of the system, the fundamental frequency of the carrier oscillator is parametrized as a vector $[o s c]=\left(f_{B}, q\right)$, where $f_{B}$ is the base frequency and $q$ is the frequency ratio, giving $f_{0}=q f_{B}$. The other parameter vectors are $[\mathrm{fbam}]=\left(\nu, \beta, w_{s}, D\right)$, interfacing the FBAM black box, and $[e n v]=(A, D, S, R)$, describing the attack/decay/sustain/release settings of the envelope 


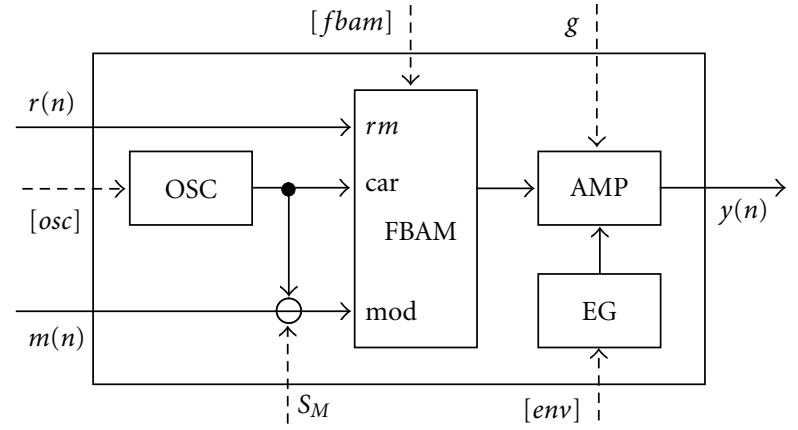

Figure 19: FBAM operator, interfaced by audio rate signals (solid) and control rate parameters (dashed).

TABLE 2: Computational load of FBAM, its variations, and related synthesis methods.

\begin{tabular}{lcccccc}
\hline Method & Figure & MUL & ADD & Scale & Total & TLU \\
\hline FBAM & 5 & 1 & 1 & 1 & 3 & 1 \\
Variation 1 & $10(\mathrm{a})$ & 1 & 2 & 1 & 4 & 1 \\
Variation 2 & $10(\mathrm{~b})$ & 1 & 2 & 1 & 4 & 1 \\
Variation 3 & $10(\mathrm{c}), 10(\mathrm{~d})$ & 2 & 1 & 1 & 4 & 2 \\
Variation 4 & $10(\mathrm{e})$ & 1 & 1 & 1 & 3 & 2 \\
Variation 5 & $10(\mathrm{f})$ & 1 & 1 & 1 & 3 & 2 \\
Variation 6 & 18 & 1 & 1 & 1 & 3 & 2 \\
PAF & & 1 & 0 & 2 & 3 & 3 \\
ModFM & & 2 & 1 & 1 & 4 & 3 \\
DSF & & 5 & 3 & 1 & 9 & 2 \\
\hline
\end{tabular}

generator EG. Parameter $g$ defines the maximum amplitude of the output $y(n)$. It should be noted that the encapsulated FBAM block may be parametrized so that the output of the operator consists only of the carrier signal produced by the OSC block.

4.2. FBAM Algorithms. Two or more FBAM operators may be arranged into multioperator configurations called FBAM algorithms. Parallel multicarrier configurations simply mix the outputs of the operators together, whereas cascaded setups connect the output of the modulator operator to the $m(n)$ and/or $r(n)$ inputs of the carrier. As a special case of the cascaded arrangement, the output of the carrier operator can be fed back to the $m(n)$ input of the modulator operator, thereby producing cross-modulated timbres. Figure 20 shows three algorithms that are used in the FBAM application examples of Section 6.

In parallel topologies, the $g$ parameter is utilized as a balance control, whereas in cascades the $g$ and the [env] vector of the modulator provide dynamic control over the spectral richness of the carrier output. The $[o s c]$ vectors define the frequency ratio between the modulator and the carrier, thus affecting the harmonic or inharmonic structure of the spectrum.

4.3. Pure Data External and Abstraction. The FBAM black box was implemented in the $\mathrm{C}$ programming language as a Pure Data (Pd) [29] external ( fbam ), which is equipped with three signal inlets, four parameter inlets, and one signal outlet. The external uses block-based processing, but supports also single-sample feedback delays by maintaining its state between successive block-based processing calls. However, sample-based cross-modulation between two operators is possible only by setting Pd's global block size to 1.

The fbam external was then patched with native Pd OSC $\sim,{ }^{*} \sim$, and $a d s r$ objects implementing the OSC, AMP, and EG blocks of the operator. The patch was interfaced with inlet and outlet ports carrying the signals and synthesis parameters discussed in Section 4.1 and encapsulated as the fbamOP Pd abstraction, which finally implements the FBAM operator.

4.4. Csound Opcode. In Csound [30], we propose a simple user-defined opcode (UDO) implementing the coefficientmodulated first-order filter, which can then be used with a variety of inputs in the various combinations discussed in this paper. It could alternatively be used inside another UDO implementing a similar structure to the Pd code in the previous section (Figure 19). The filter UDO is isolated from the other parts of the code for efficiency reasons: it requires a processing vector of one sample for the feedback to be implemented. As this is computationally costly, it is separated from the rest of the code, as shown in Listing 1.

With this in hand, the basic FBAM algorithm is implemented with the code presented in Listing 2.

\section{Evaluation}

This section evaluates the FBAM method by comparing it to related nonlinear synthesis techniques. First the basic FBAM equation is contextualized in the parameter spaces of the related techniques, and then the computational load of the methods are compared.

5.1. Relation to Existing Nonlinear Distortion Methods. Equation (21) reconstructed the basic FBAM waveform using a hybrid amplitude and phase modulation technique. By ignoring the phase modulation component $\phi(n)$ in (21), one can look at the effect of AM separately (see Figure 21(a)). On the other hand, by setting the $A(n)$ component to unity, one can see the effect of the isolated phase modulation operation, as shown in Figure 21(b). As can be seen, the FBAM output is mostly determined by the AM operation between a complex signal and a sinusoid and the effect of phase modulation in (21) is minor.

This suggests that FBAM is more related to the ringmodulation-based PAF [17] and the recent ModFM [18, 19] methods than it is to the classic FM [11] synthesis technique. Figure 22 compares the waveform and spectrum of FBAM (dashed), PAF (solid), and ModFM (dots). The parameters were chosen by first selecting the maximum $\beta$ value according to the criteria of Figure 7, then normalizing the magnitude of the fundamental to $0 \mathrm{~dB}$, finding the highest frequency component above the $-100 \mathrm{~dB}$ threshold, 


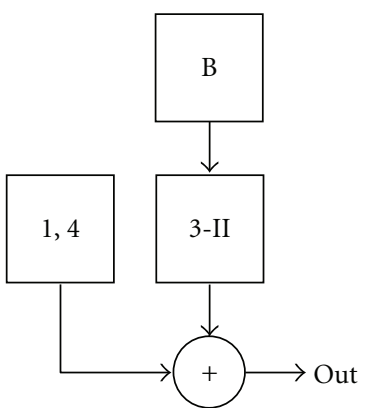

(a)

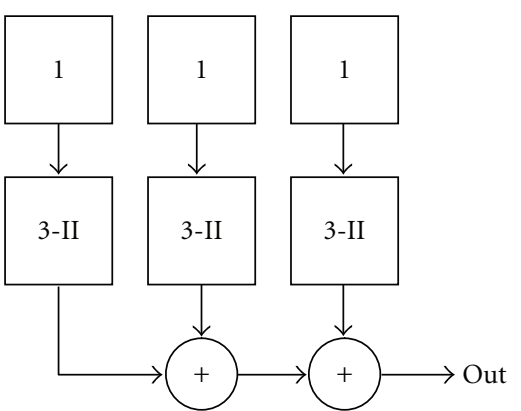

(b)

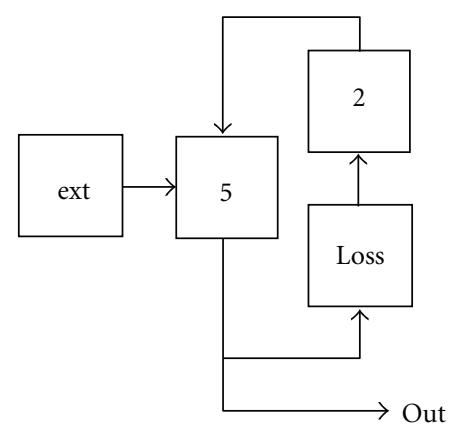

(c)

FIgURE 20: FBAM algorithms for (a) subtractive, (b) formant, and (c) abstract physical modeling sound synthesis arrangements. Variation numbers are given inside the operator boxes (B denotes the basic FBAM equation).

and finally matching the magnitudes of the highest partials. The waveform maxima in Figure 22(a) have been timealigned for easy comparison, showing that the waveform peak is the narrowest in ModFM and widest in PAF. This is reflected in the spectrum plot of Figure 22(b), where the spectral slope of FBAM is less accented than the exponential decay of PAF, but steeper than the one produced by ModFM.

The DSF formulation of PAF is similar to (4) given in [16]. A related closed form approximation of FBAM is given by

$$
y(n)=\frac{\cos \left(\omega_{0} n\right)}{\left[1-2 a \cos \left(\omega_{0} n\right)+a^{2}\right]^{3}},
$$

which is similar to Equation (593) of [31], in which the ratio parameter $a$ is dependent on the $\beta$ and $\omega_{0}$ parameters of FBAM. The waveform and spectrum of (44) are shown in Figures 23(a) and 23(b), while its normalized waveshaper formulation is plotted in Figure 23(c).

As expected, a waveshaper based on Chebyshev's polynomials [1] can reproduce the magnitude spectrum of FBAM exactly when $\beta=1$. Its transfer function takes an exponential shape with a close match to the waveshaper shown in Figure 23(c).

5.2. Computational Load. Table 2 summarizes the number of multiplications (MUL, Scale), additions (ADD), and table lookups (TLU) for the basic FBAM, its variations, and related synthesis techniques per output sample. Each oscillator involved in the algorithm is counted as a table-lookup operation. It is noted that FBAM, PAF, and ModFM are close to each other in the total number of simple operations, but that FBAM has the benefit of not using precomputed lookup tables in the complex modulator signal evaluation. This is an advantage of FBAM, because interpolating table lookups are considerably more resource consuming than simple addition and multiplication operations. DSF gains the widest output bandwidth at the cost of additional operations per output sample.

\begin{tabular}{|ll|}
\hline opcode Cmf, a, aak & \\
& setksmps 1 \\
ay & init 0 \\
asig, amod, kb & xin \\
ay & asig + amod*ay*kb $^{*}$ ay \\
endop & \\
\hline
\end{tabular}

Listing 1: Csound UDO for the coefficient-modulated first-order IIR filter.

\section{Applications}

The brief discussion of FBAM applications in the authors' earlier work is now extended, and the sonic palette of FBAM is expanded further with three novel applications. Additional application examples are available at the accompanying web page [15].

6.1. Subtractive Synthesis without Resonant Filters. FBAM can imitate classic analog waveforms with variations 1 and 4 . Although the spectral brightness in these cases is limited (see Figures 11,15, and 16), it may be conveniently shaped with the $\beta$ parameter. A single FBAM operator can, therefore, function as a simple low-cost subtractive synthesizer that implements both the source oscillator and the low-pass filter of a virtual analog system. This basic scheme can be further refined by simulating the resonance characteristics of the low-pass filter with (32). The structure of such a setup is shown in Figure 20(a).

A related DSF-based implementation [32], without resonance control, requires 25 operations per output sample for the direct closed form evaluation and additional control rate operations for dynamic cut-off frequency updates (whereas FBAM uses 5 operations in total). However, the spectral brightness of the DSF-based method is considerably higher and, at the same time, bandlimited.

FBAM can control the amount of aliasing by scaling $\beta$ with the curve shown in Figure 7, while the bandwidth of 


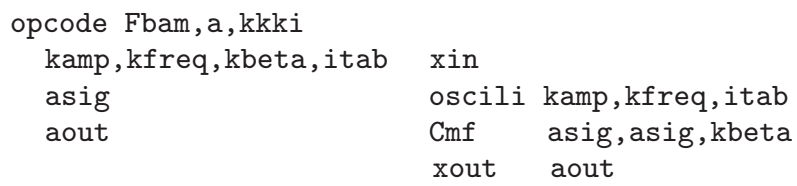

endop

LIsTING 2: Csound UDO for the basic FBAM algorithm.

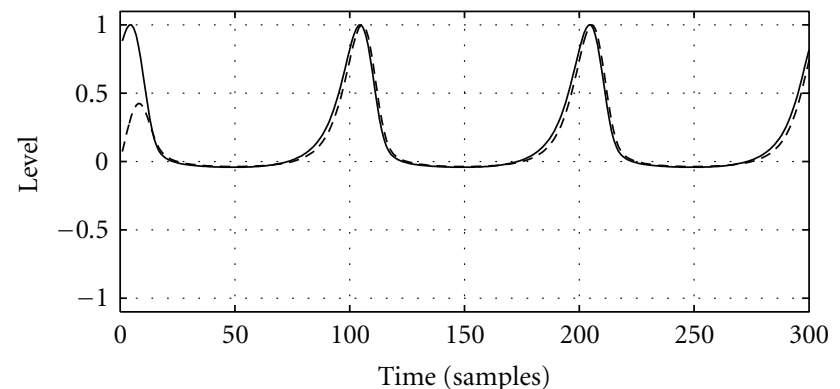

(a)

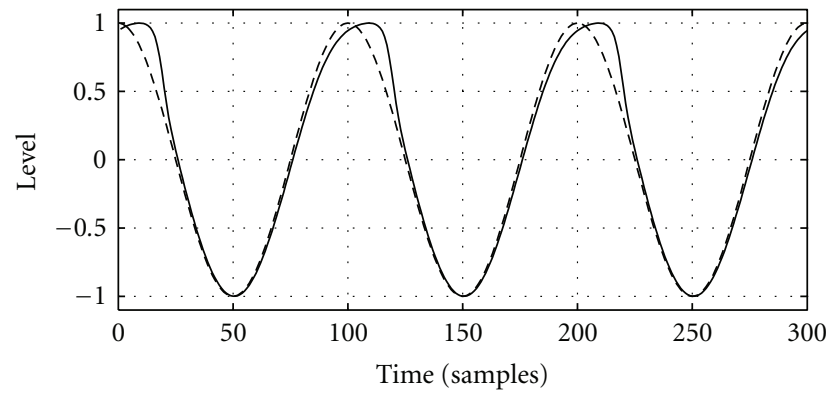

(b)

FIgURE 21: (a) FBAM waveform (dashed) and reconstructed using AM (solid). (b) Cosine waveform (dashed) and FBAM reconstruction using PM (solid). Signals were generated with $\beta=1$ and $f_{0}=500 \mathrm{~Hz}$.

FBAM can be increased by oversampling. However, a more cost-effective bandwidth extension is achieved by adding partials to the modulator signal, for example, by raising the modulator to the third power, yielding $\cos ^{3}\left(\omega_{0} n\right)=$ $0.75 \cos \left(\omega_{0} n\right)+0.25 \cos \left(3 \omega_{0} n\right)$. Figure 24 compares the waveform and spectrum of this extended bandwidth modification to the basic FBAM output, using $\beta$ values that keep the aliasing level $-100 \mathrm{~dB}$ from the maximum. Note that the cubed cosine modulator permits higher $\beta$ values because $\left|\cos \left(\omega_{0} n\right)\right| \leq 1$. The cost of this modification is two multiplications per output sample.

6.2. Formants. FBAM can synthesize formant-based timbres with parallel resonator stacks, as shown in Figure 20(b). In this algorithm, each modulator operator is tuned to the center frequency of the formant, while all carriers are tuned to the fundamental frequency of the tone (possibly with slight detuning for the added vibrato, as suggested by [33],

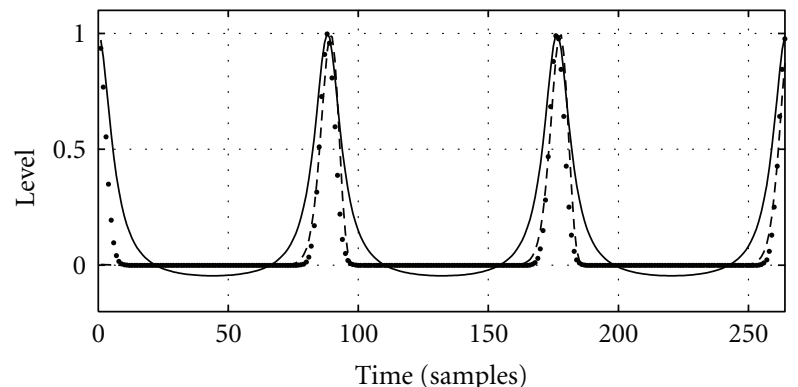

(a)

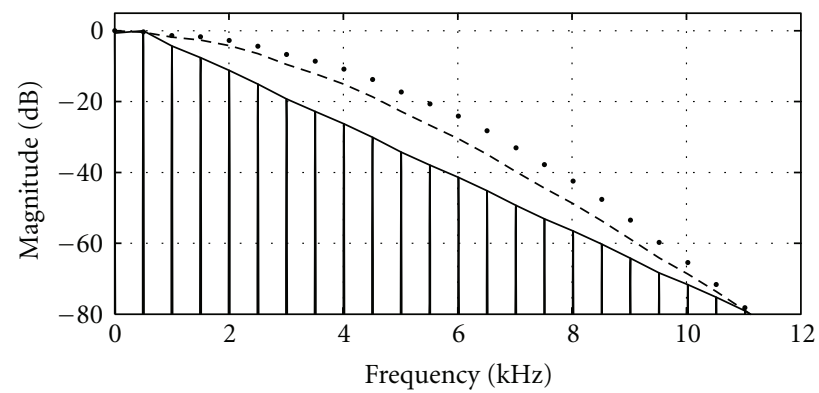

(b)

Figure 22: Waveform and spectrum of $\operatorname{FBAM}(\beta=1.62$, dashed), PAF (bandwidth $\delta=0.164$, solid), and ModFM ( $k=25$, dotted). Signals were generated with $f_{c}=f_{m}=500 \mathrm{~Hz}$.

for vocal timbres). Carrier $\beta$ and $g$ values control the width and relative amplitude of the formant.

Figure 25 shows an example FBAM vocal timbre, with fundamental frequency $f_{0}=196 \mathrm{~Hz}$ and approximate formants at $f_{1}=700 \mathrm{~Hz}, f_{2}=1090 \mathrm{~Hz}$, and $f_{3}=$ $2700 \mathrm{~Hz}$. Because the formant frequencies do not coincide with the harmonic series of the fundamental, each formant is modeled using the double-carrier formula of (32). For example, formant $f_{3}$ is modeled using harmonic ordinals $k_{\mathrm{LO}}=\operatorname{int}\left(f_{3} / f_{0}\right)=13$ and $k_{\mathrm{HI}}=k_{\mathrm{LO}}+1=14$, giving $f_{\mathrm{LO}}=13 f_{0}$ and $f_{\mathrm{HI}}=14 f_{0}$, thereby coinciding with the harmonic spectra of the fundamental $f_{0}$. The modulator is formed as a weighted sum of two cosine oscillators, which are tuned to frequencies $f_{\mathrm{LO}}$ and $f_{\mathrm{HI}}$ and weighted using linear interpolation as $g_{\mathrm{HI}}=f_{3} / f_{0}-k_{\mathrm{LO}}$ and $g_{\mathrm{LO}}=1-g_{\mathrm{HI}}$. The bandwidth of formant 3 is controlled by setting the modulation index of the carrier $\beta_{3}=0.6$, and, finally, the operator output is finally scaled $35 \mathrm{~dB}$ below formants 1 and 


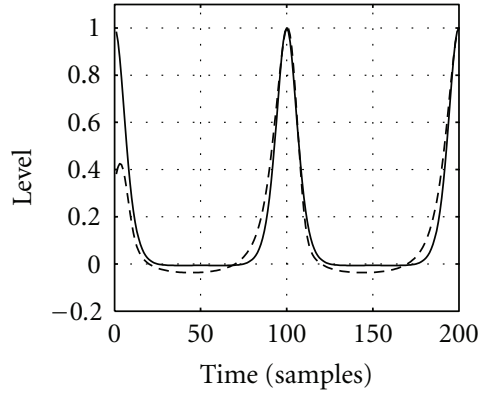

(a)

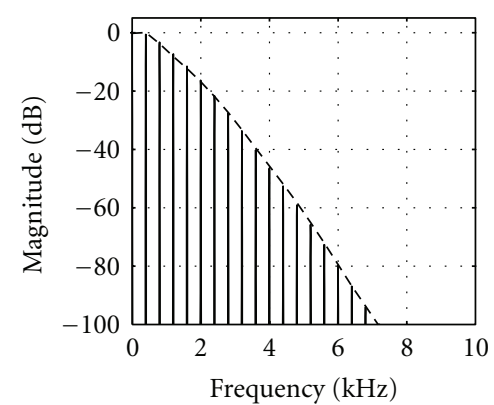

(b)

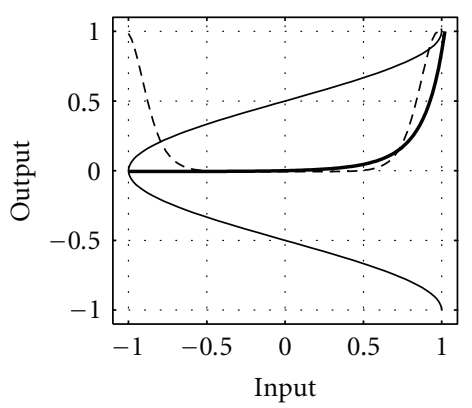

(c)

FIgURE 23: (a,b) Closed-form approximation ( $a=2.5$, solid) of FBAM ( $\beta=1$, dashed) and (c) its waveshaper formulation (input as thin, shaping function as thick, and output as dashed line).

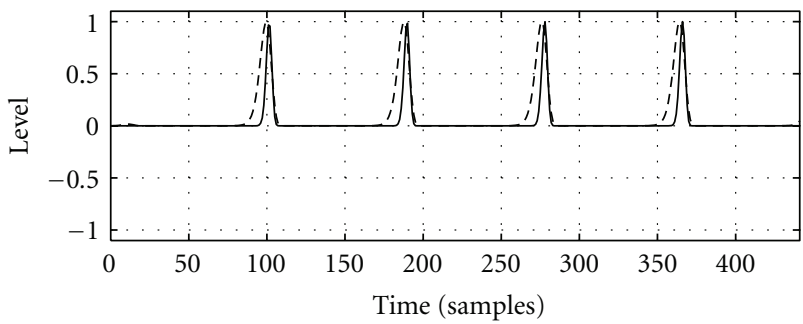

(a)

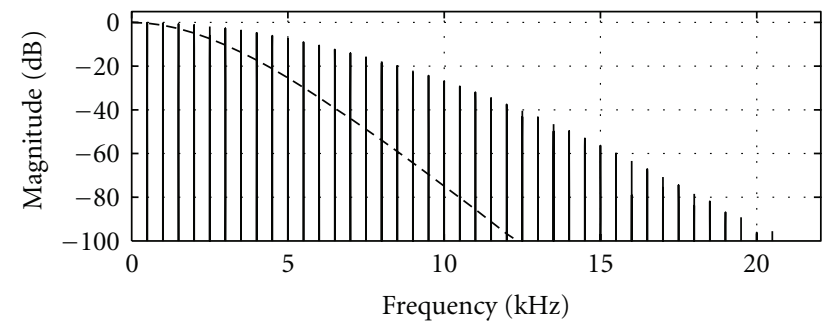

(b)

FIGURE 24: Extended bandwidth FBAM (cubed cosine modulator with $\beta=5.8$, solid) and basic FBAM ( $\beta=1.5$, dashed), with $f_{0}=500 \mathrm{~Hz}$.

2. A similar dynamic procedure facilitates smooth morphing between different vowel sounds.

6.3. Abstract Physical Modeling Synthesis. One-dimensional digital waveguide implementations, such as simple plucked string models, are often based on a non-interpolating delay line that is cascaded with a fine-tuning fractional-delay filter $[24,34,35]$. Since FBAM variation 5 embodies a non-interpolating delay line internally and since variation 2 is able to realize the fine-tuning fractional delay in form of a first-order allpass structure [36], these two FBAM operators may be arranged into a looped topology similar to that of a digital waveguide. A feedback path loss filter (which can be implemented, for example, as a simple two-point moving averager) completes this simple model. The topology of such a system is shown in Figure 20(c). The excitation (ext) of the system may be generated by a high-energy FBAM operator, which facilitates tailored excitation signals, or by an external excitation source.

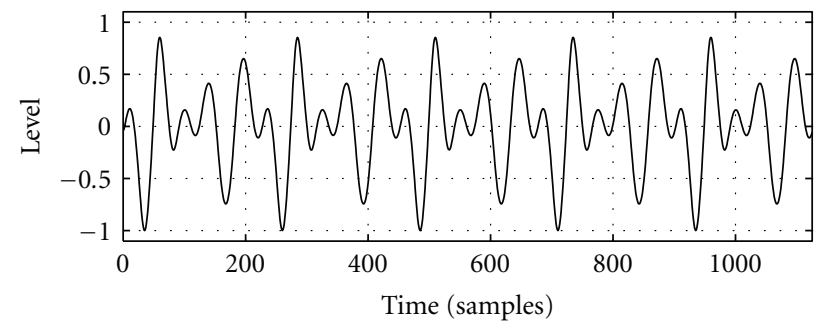

(a)

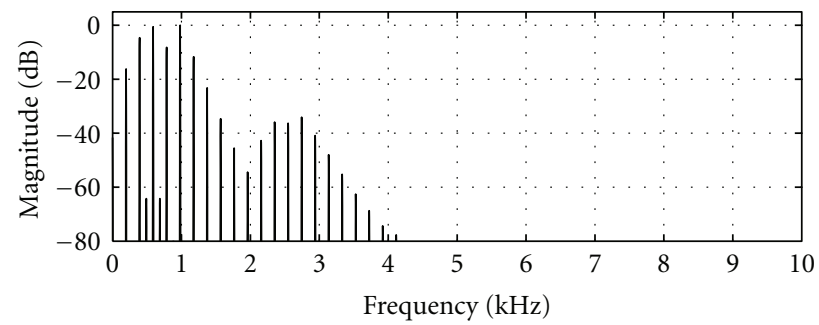

(b)

FIGURE 25: Waveform and spectrum of FBAM vocal timbre ( $a$ : $)$ with three formants $\left(f_{0}=196 \mathrm{~Hz}\right)$.

In this structure, the coefficients of the delay line operator, the allpass operator, or both may be modulated. Figure 26 shows the spectrogram of the output signal of an allpass modulated FBAM algorithm that was excited with an external noise source. The modulating frequency was slightly detuned from the half length of the delay line, producing the thick dispersive spring-like beating effect discussed in [37].

We call this fusion of physical modeling and abstract sound synthesis techniques as Abstract Physical Modeling Synthesis, which is a potential source of interesting timbres. However, stability and tuning problems need to be resolved before the method becomes usable in practical scenarios: the presented system becomes unstable with large $\beta$ values when the modulator period is an exact fractional multiple of the period of the delay line, that is, $P_{\bmod }=P_{\text {delay }} k / 4$, where $k$ is an integer. The tuning problems appear because the total delay of the system depends on the allpass operator coefficient. 


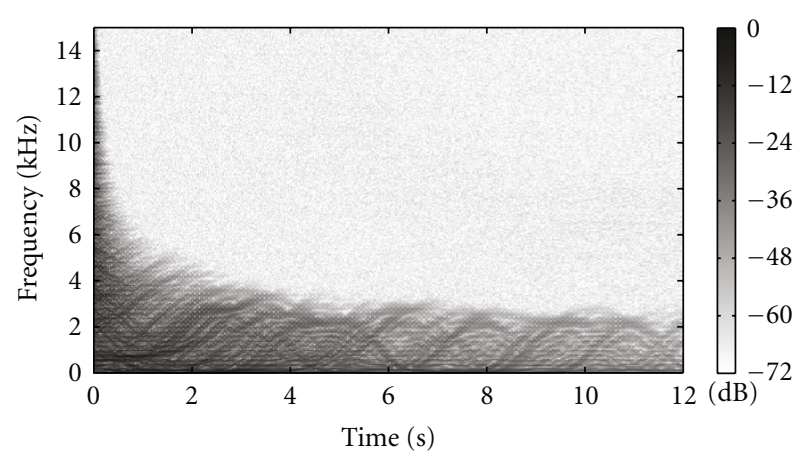

Figure 26: FBAM as an abstract physical model $\left(f_{x}=130 \mathrm{~Hz}, f_{m}\right.$ : $f_{x}=0.498, \beta=0.9$ ).

6.4. Digital Audio Effects. By employing an arbitrary input signal instead of an oscillator, the FBAM algorithm becomes a digital audio effect. The characteristics of this effect bear some semblance to other adaptive distortion processes such as AdFM [27], Adaptive SpSB [38], and Adaptive Phase Distortion [10]. In this class of effects, new partials are added to the spectrum, producing a component-rich output. The modulation index $\beta$ can be used to control the amount of distortion (and new partials).

As discussed in Section 3.6.1, there are two main methods of implementing such an adaptive effect, by employing the input as a modulator (self-modulation) or by employing a separate oscillator (generally sinusoidal) whose frequency can be controlled by pitch-tracking the input signal. In the former, the carrier-modulator frequency ratio is locked in a $1: 1$ proportion, whereas in the latter, various extra effects can be produced by modifying this ratio, for instance, the generation of inharmonic spectra. In addition, this method has some more controlled means of timbral modification, as the modulation source will contain a small number of components leading to a more predictable spectrum.

Figure 27 shows a comparison between the steady-state spectra of a flute tone and its adaptive FBAM-processed version. For this example, a carrier-modulation ratio of $1: 2.4$ and $\beta=0.7$ was used to produce an inharmonic spectrum reminiscent of multiphonic sound. This effect was implemented using a cosine modulator whose frequency was determined by a pitch tracker applied to the input signal.

\section{Conclusion}

This work investigated the feedback amplitude modulation principle and its variations for sound synthesis purposes. The FBAM synthesis appears to be a promising synthesis method, which has not been fully explored previously, although the basic idea has been known for a long time. The method has similarities to the existing nonlinear distortion synthesis techniques, such as the creation of rich spectra based on one or a few sinusoidal input signals. Furthermore, the spectral brightness can be easily controlled using a single parameter, which is comparable to the well-known modulation index in FM synthesis. In FBAM, the corresponding parameter is the feedback gain, introduced here as the $\beta$ parameter. It

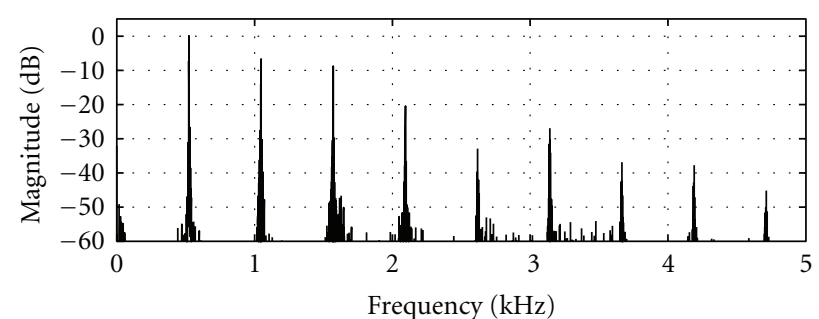

(a)

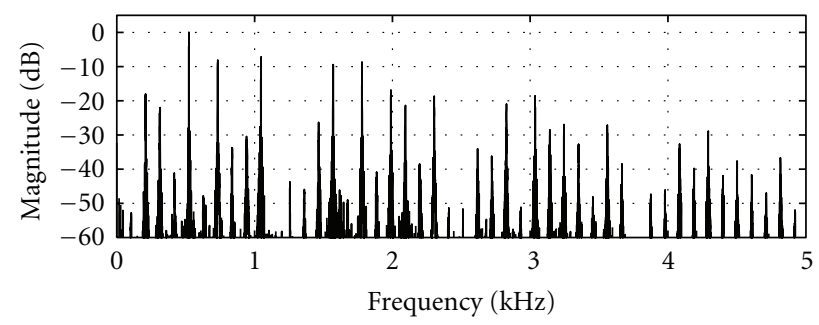

(b)

Figure 27: Spectra of (a) C4 flute tone and (b) its Adaptive FBAMprocessed version, with the carrier-modulator ratio set to $1: 2.4$ and $\beta=0.7$.

is important to note, however, that the details of spectral evolution are different from that of FM synthesis, for example, because of the absence of holes in the spectrum, which are a typical feature of FM synthesis.

This work continued from the authors' previous study [2] in which the basic FBAM system was defined as a nonlinear generalization of the one-pole digital filter. The present work applied the periodically linear time-varying (PLTV) digital filter theory and derived the time-varying frequency response of the system. This was validated by the amplitude and phase modulation implementation of the FBAM algorithm based on the magnitude and phase response of the time-varying filter. This enabled defining the limits of stability for the system as well as obtaining a method for normalizing its output.

Six variations to this basic scheme were then refined. The first variation adds a delayed input signal inside the feedback loop, thus varying mainly the waveform without affecting much the overall signal spectrum. Variation 2 is reminiscent of an allpass filter structure, and the first harmonic dominates in the output spectrum with most parameter settings. Two different useful versions of variation 3 were found, involving a second modulation signal. This enables creating a formant in the output signal spectrum.

Variation 4 derived from the basic FBAM contains a nonlinear waveshaping function in the feedback loop. Experiments with several choices of the waveshaper show interesting properties for some of them. For example, with a full-wave rectifier (i.e., the ABS function), the output signal of variation 4 is composed only of odd harmonics of the input signal, thus enabling the creation of square wave-like signals. Variation 5 generalizes the feedback delay with a delay line of arbitrary length. This leads to a second control over the spectral brightness with the delay-line length parameter. Finally, this paper also demonstrates that, for 
some uses, it is interesting to generalize the FBAM algorithm as a coefficient-modulated IIR filter. This allows, among other things, the development of a novel digital audio effect derived from the original synthesis algorithm.

For interesting musical applications, the FBAM operator abstraction was refined and its use in various programming environments was discussed. Additionally, a separation of the input and modulation signals in the FBAM oscillator was proposed to enable arbitrary modular systems built out of several elementary FBAM structures. Now, two or more FBAM oscillators can be cascaded.

The FBAM method was finally evaluated in comparison to other established distortion synthesis techniques. A closed-form formula for the FBAM output waveform was presented and its corresponding waveshaping transfer function discussed. The computational load of FBAM was analyzed and demonstrated to compare favorably with PAF and ModFM techniques. This article concluded by demonstrating some applications for FBAM and its variations, including a modification for the bandwidth extension. A logical future extension of this work would exploit the theoretical framework of Section 2 in the case of second- and higher-order feedback systems.

Sound examples and software are available at [15].

\section{Acknowledgments}

This work has been supported by the EU 7th Framework Programme (the SAME project, ref. 215749) and by the Academy of Finland (Project no. 122815). This is a revised and extended version of the paper entitled Five Variations on a Feedback Theme published in the Proceedings of the 12th International Conference on Digital Audio Effects (DAFx09), Como, Italy, September 2009 [2].

\section{References}

[1] C. Dodge and T. Jerse, Computer Music: Synthesis, Composition, and Performance, Schirmer Books, New York, NY, USA, 1985.

[2] V. Lazzarini, J. Timoney, J. Kleimola, and V. Välimäki, "Five variations on a feedback theme," in Proceedings of the 12th International Conference on Digital Audio Effects (DAFx '09), pp. 139-145, Como, Italy, September 2009.

[3] J.-C. Risset, An Introductory Catalog of Computer-Synthesized Sounds, Bell Laboratories, 1969, Published in 1995 as part of The Historical CD of Digital Sound Synthesis, Computer Music Currents 13, Wergo WER 20332.

[4] A. Layzer, "Some idiosyncratic aspects of computer synthesized sound," in Proceedings of the Annual Conference American Society of University Composers, pp. 27-39, 1971.

[5] N. Valsamakis and E. R. Miranda, "Iterative sound synthesis by means of cross-coupled digital oscillators," Digital Creativity, vol. 16, no. 2, pp. 90-98, 2005.

[6] M. Cherniakov, V. I. Sizov, and L. Donskoi, "Synthesis of a periodically time-varying digital filter," IEE Proceedings: Vision, Image and Signal Processing, vol. 147, no. 5, pp. 393399, 2000.

[7] M. Cherniakov, An Introduction to Parametric Digital Filters and Oscillators, John Wiley \& Sons, New York, NY, USA, 2003.
[8] J. Pekonen, "Coefficient modulated first-order allpass filter as a distortion effect," in Proceedings of the 11th Conference on Digital Audio Effects (DAFx '08), pp. 83-87, Espoo, Finland, 2008.

[9] J. Timoney, V. Lazzarini, J. Pekonen, and V. Välimäki, “Spectrally rich phase distortion sound synthesis using an allpass filter," in Proceedings of the IEEE International Conference on Acoustics, Speech, and Signal Processing (ICASSP '09), pp. 293296, Taipei, Taiwan, April 2009.

[10] J. Timoney, V. Lazzarini, V. Välimäki, and J. Pekonen, "Adaptive phase distortion synthesis," in Proceedings of the 12th International Conference on Digital Audio Effects (DAFx '09), Como, Italy, September 2009.

[11] J. M. Chowning, "The synthesis of complex audio spectra by means of frequency modulation," Journal of the Audio Engineering Society, vol. 21, no. 7, pp. 526-534, 1973.

[12] K. Steiglitz, A Digital Signal Processing Primer with Applications to Digital Audio and Computer Music, Addison-Wesley, Reading, Mass, USA, 1996.

[13] J. Laroche, "On the stability of time-varying recursive filters," Journal of the Audio Engineering Society, vol. 55, no. 6, pp. 460471, 2007.

[14] D. Benson, Music: A Mathematical Offering, Cambridge University Press, Cambridge, UK, 2006.

[15] FBAM, http://www.acoustics.hut.fi/go/jasp-fbam.

[16] J. A. Moorer, "The synthesis of complex audio spectra by means of discrete summation formulae," Journal of the Audio Engineering Society, vol. 24, no. 9, pp. 717-727, 1976.

[17] M. Puckette, "Formant-based audio synthesis using nonlinear distortion," Journal of the Audio Engineering Society, vol. 43, no. 1-2, pp. 40-47, 1995.

[18] V. Lazzarini and J. Timoney, "New methods of formant analysis-synthesis for musical applications," in Proceedings of the International Computer Music Conference (ICMC '09), Montreal, Canada, August 2009.

[19] V. Lazzarini and J. Timoney, "Theory and practice of modified frequency modulation synthesis," Journal of the Audio Engineering Society, vol. 58, no. 6, pp. 459-471, 2010.

[20] M. Le Brun, "Digital waveshaping synthesis," Journal of the Audio Engineering Society, vol. 27, no. 4, pp. 250-266, 1979.

[21] D. Arfib, "Digital synthesis of complex spectra by means of multiplication of nonlinear distorted sine waves," Journal of the Audio Engineering Society, vol. 27, no. 10, pp. 757-768, 1979.

[22] N. Tomisawa, "Tone production method for an electronic musical instrument," US patent 4,249,447, 1979.

[23] M. Mathews, "MUSIC V, FORTRAN source code," in The Audio Programming Book, R. Boulanger and V. Lazzarini, Eds., MIT Press, Cambridge, Mass, USA, 2010.

[24] T. I. Laakso, V. Välimäki, M. Karjalainen, and U. K. Laine, "Splitting the unit delay: tools for fractional delay filter design,” IEEE Signal Processing Magazine, vol. 13, no. 1, pp. 30-60, 1996.

[25] B. Schottstaedt, "The simulation of natural instrument tones using frequency modulation with a complex modulating wave," Computer Music Journal, vol. 1, no. 4, pp. 46-50, 1977.

[26] M. LeBrun, "A derivation of the spectrum of FM with a complex modulating wave," Computer Music Journal, vol. 1, no. 4, pp. 51-52, 1977.

[27] V. Lazzarini, J. Timoney, and T. Lysaght, "The generation of natural-synthetic spectra by means of adaptive frequency modulation," Computer Music Journal, vol. 32, no. 2, pp. 9-22, 2008. 
[28] J. Chowning and D. Bristow, FM Theory and Applications, Hal Leonard, Winona, Minn, USA, 1987.

[29] M. Puckette, The Theory and Technique of Electronic Music, World Scientific Press, River Edge, NJ, USA, 2007.

[30] R. Boulanger, The Csound Book: Perspectives in Software Synthesis, Sound Design, Signal Processing, and Programming, MIT Press, Cambridge, UK, 2000.

[31] L. B. W. Jolley, Summation of Series, Dover, New York, NY, USA, 1961.

[32] J. Lazzaro and J. Wawrzynek, "Subtractive synthesis without filters," in Audio Anecdotes II-Tools, Tips, and Techniques for Digital Audio, pp. 55-64, A. K. Peters, Ltd, Wellesley, Mass, USA, 2004.

[33] J. Chowning, "Frequency modulation synthesis of the singing voice," in Current Directions in Computer Music Research, M. Mathews and J. R. Pierce, Eds., pp. 57-63, MIT Press, Cambridge, Mass, USA, 1989.

[34] J. O. Smith, "Physical modeling using digital waveguides," Computer Music Journal, vol. 16, no. 4, pp. 74-87, 1992.

[35] V. Välimäki, J. Huopaniemi, M. Karjalainen, and Z. Jánosy, "Physical modeling of plucked string instruments with application to real-time sound synthesis," Journal of the Audio Engineering Society, vol. 44, no. 5, pp. 331-353, 1996.

[36] D. A. Jaffe and J. O. Smith, "Extensions of the Karplus-Strong plucked-string algorithm," Computer Music Journal, vol. 7, no. 2, pp. 56-69, 1983.

[37] J. Kleimola, J. Pekonen, H. Penttinen, V. Välimäki, and J. Abel, "Sound synthesis using an allpass filter chain with audio-rate coefficient modulation," in Proceedings of the 12th International Conference on Digital Audio Effects (DAFx '09), pp. 305-312, Como, Italy, September 2009.

[38] V. Lazzarini, J. Timoney, and T. Lysaght, "Nonlinear distortion synthesis using the split-sideband method, with applications to adaptive signal processing," Journal of the Audio Engineering Society, vol. 56, no. 9, pp. 684-695, 2008. 